\title{
Viscous Flow Around a Rigid Body Performing a Time-periodic Motion
}

\author{
Thomas Eiter(D) and Mads Kyed \\ Communicated by Y. Shibata
}

\begin{abstract}
The equations governing the flow of a viscous incompressible fluid around a rigid body that performs a prescribed time-periodic motion with constant axes of translation and rotation are investigated. Under the assumption that the period and the angular velocity of the prescribed rigid-body motion are compatible, and that the mean translational velocity is non-zero, existence of a time-periodic solution is established. The proof is based on an appropriate linearization, which is examined within a setting of absolutely convergent Fourier series. Since the corresponding resolvent problem is ill-posed in classical Sobolev spaces, a linear theory is developed in a framework of homogeneous Sobolev spaces.
\end{abstract}

Mathematics Subject Classification. 35Q30, 35B10, 76D05, 76D07, 76U05.

Keywords. Navier-Stokes, Oseen flow, Time-periodic solutions, Rotating obstacles.

\section{Introduction}

We investigate the fluid flow past a rigid body $\mathcal{B}$ that moves through an infinite three-dimensional liquid reservoir with prescribed velocity

$$
V(t, x)=\xi(t)+\eta \wedge\left(x-x_{\mathrm{C}}(t)\right)
$$

with respect to its center of mass $x_{\mathrm{C}}$. Here $t \in \mathbb{R}$ and $x \in \mathbb{R}^{3}$ denote time and spatial variable, respectively, $\xi:=\frac{\mathrm{d}}{\mathrm{d} t} x_{\mathrm{C}}$ is the translation velocity and $\eta$ the angular velocity of $\mathcal{B}$ with respect to its center of mass. We only consider the case where the angular velocity $\eta$ is constant, but the translation velocity $\xi$ may depend on time. In a frame attached to the body, with origin at its center of mass $x_{\mathrm{C}}$, the motion of an incompressible Navier-Stokes fluid around $\mathcal{B}$ that adheres to $\mathcal{B}$ at the boundary is described by the equations

$$
\left\{\begin{aligned}
\rho\left(\partial_{t} u+\eta \wedge u-\eta \wedge x \cdot \nabla u-\xi \cdot \nabla u+u \cdot \nabla u\right)=f+\mu \Delta u-\nabla \mathfrak{p} & \text { in } \mathbb{R} \times \Omega, \\
\operatorname{div} u=0 & \text { in } \mathbb{R} \times \Omega, \\
u=\xi+\eta \wedge x & \text { on } \mathbb{R} \times \partial \Omega \\
\lim _{|x| \rightarrow \infty} u(t, x)=0 & \text { for } t \in \mathbb{R}
\end{aligned}\right.
$$

see [12, Section 1]. Here $\Omega:=\mathbb{R}^{3} \backslash \overline{\mathcal{B}}$ is the exterior domain surrounding $\mathcal{B}$, and $\mathbb{R}$ represents the time axis. The functions $u: \mathbb{R} \times \Omega \rightarrow \mathbb{R}^{3}$ and $\mathfrak{p}: \mathbb{R} \times \Omega \rightarrow \mathbb{R}$ describe velocity and pressure fields of the fluid. The constants $\rho>0$ and $\mu>0$ denote density and viscosity, respectively. For the sake of generality, we additionally consider an external body force $f: \mathbb{R} \times \Omega \rightarrow \mathbb{R}^{3}$.

In this paper, we investigate a configuration where the rigid body $\mathcal{B}$ translates periodically with some prescribed time period $\mathcal{T}>0$. More precisely, we assume the data

$$
\xi(t+\mathcal{T})=\xi(t), \quad f(t+\mathcal{T}, x)=f(t, x)
$$

to be $\mathcal{T}$-time-periodic As the main theorem we show existence of a solution $(u, \mathfrak{p})$ to (1.1) that shares this time periodicity. 
We consider a prescribed motion of $\mathcal{B}$ where the axes of translation and rotation do not vary over time and are parallel. Without loss of generality, both are directed along the $x_{1}$-axis such that

$$
\xi(t)=\alpha(t) \mathrm{e}_{1}, \quad \eta=\omega \mathrm{e}_{1}
$$

for some $\mathcal{T}$-periodic function $\alpha: \mathbb{R} \rightarrow \mathbb{R}$ and a constant $\omega \in \mathbb{R}$. Note that, at least in the case where $\xi$ is time-independent, this assumption can be made without loss of generality as long as $\xi \cdot \eta \neq 0$ due to the Mozzi-Chasles theorem.

We assume that the mean translational velocity of the body over one time period is non-zero:

$$
\lambda:=\frac{1}{\mathcal{T}} \int_{0}^{\mathcal{T}} \alpha(t) \mathrm{d} t \neq 0 .
$$

The case of vanishing mean translational velocity shall not be treated here. Not only does the fluid flow exhibit different physical properties when (1.2) is not satisfied, due to the absence of a wake region in this case, but also the mathematical properties of the linearization of (1.1) differ significantly. If (1.2) is satisfied, the linearization of (1.1) is a time-periodic generalized Oseen system, for which we shall establish suitable $\mathrm{L}^{q}$ estimates in order to show existence of a solution to (1.1). If (1.2) is not satisfied, the linearization of (1.1) is a time-periodic generalized Stokes system, for which similar estimates cannot be derived. In this case, problem (1.1) thus has to be approached in a different way, which has recently been done by Galdi [15].

Since the case $\eta=0$ was treated in [18], we only consider the case $\eta \neq 0$ in the following. Observe that then $\eta \wedge x \cdot \nabla$ is a differential operator with unbounded coefficient. Therefore, the linearization of (1.1) cannot be treated as a lower-order perturbation of the time-periodic Oseen problem, even if $\eta$ is "small". In particular, as we will see below, the corresponding resolvent problem also requires an analysis in a different functional setting. This observation reflects the properties of the corresponding stationary problem (see [13, Chapter VIII]), which can be regarded as a special case of the time-periodic problem. In order to find a framework in which the time-periodic generalized Oseen problem is well posed, we employ the idea from $[16,17]$, where the steady-state problem corresponding to (1.1) was considered, and the rotation term $\eta \wedge u-\eta \wedge x \cdot \nabla u$ was handled by a change of coordinates into a non-rotating frame. This procedure only yields suitable estimates for time-periodic solutions when the change of coordinates maintains the time periodicity of the involved functions. This is the case if the angular velocity $\omega$ is an integer multiple of the angular frequency $2 \pi / \mathcal{T}$ of the time-periodic data. For simplicity, we assume

$$
\omega=2 \pi / \mathcal{T} \text {. }
$$

This condition means that during one period the rigid body completes one full revolution. In other words, the rotation and the time-periodic data, which may be regarded as two different sources of time-periodic forcing, have to be compatible.

The equations governing the fluid flow around a rigid body that performs a prescribed rigid motion have been studied by many researchers during the last decades. The first successful attempts of a rigorous mathematical treatment date back to the fundamental works of Oseen [44], LERAY [36,37] and Ladyžhenskaya [34,35]. The study of time-periodic Navier-Stokes flows was proposed in a short note by Serrin [47], which induced Prodi [45], Yudovich [56] and Prouse [46] to initiate the examination in bounded domains. Through the years, this investigation has been continued and extended to other types of domains and fluid-flow configurations by several authors; see for example $[5,10,11,14,18,21-$ 23,28-31,33,38-43,49,51-55]. We refer to [19] for a more detailed overview. Concerning in particular time-periodic Navier-Stokes flows around rigid bodies, more specifically the three-dimensional exteriordomain configuration, we emphasize the fundamental work of Yamazaki [55], who introduced a setting of $\mathrm{L}^{3, \infty}(\Omega)$ spaces to obtain time-periodic solutions in the case $\xi=\eta=0$. The main estimates in [55] are based on well-known $\mathrm{L}^{p}-\mathrm{L}^{q}$ estimates of the Stokes semigroup. If one replaces these estimates with the $\mathrm{L}^{p}-\mathrm{L}^{q}$ estimates obtained by Shibata [48] for an Oseen semigroup with rotational effects, the approach in [55] also seems to yield existence of time-periodic solutions to (1.1) in an $\mathrm{L}^{3, \infty}(\Omega)$ framework in the case of constant non-zero parameters $\xi \neq 0, \eta \neq 0$. This analysis was recently carried out by Geissert, Hieber 
and Nguyen [23], who introduced a general semigroup-based approach to show existence of mild solutions to time-periodic problems. Using a recent result by Hishida [27], who established $\mathrm{L}^{p}$-L $\mathrm{L}^{q}$ estimates for an evolution operator corresponding to a linearization of the Navier-Stokes equations in the case of time-dependent $\xi(t)$ and $\eta(t)$, the approach of Yamazaki [55] even leads to time-periodic solutions in an $\mathrm{L}^{3, \infty}(\Omega)$ framework for general time-periodic $\xi(t)$ and $\eta(t)$. In this general case, Galdi and Silvestre [21] already established the existence of time-periodic solutions in an $\mathrm{L}^{2}(\Omega)$ setting via a Galerkin approach.

As the main novelty of the present paper, we establish existence of strong solutions to (1.1) in an $\mathrm{L}^{q}(\Omega)$ setting for a certain range of exponents $q \in(1, \infty)$. In this setting, better information on the spatial decay of the solutions can be derived compared to the $\mathrm{L}^{3, \infty}(\Omega)$ and $\mathrm{L}^{2}(\Omega)$ frameworks described above. Our approach is based on an analysis of the linearization of (1.1) and the associated resolvent problem

$$
\left\{\begin{aligned}
i s v+\omega\left(\mathrm{e}_{1} \wedge v-\mathrm{e}_{1} \wedge x \cdot \nabla v\right)-\Delta v-\lambda \partial_{1} v+\nabla p=F & \text { in } \Omega, \\
\operatorname{div} v=0 & \text { in } \Omega, \\
v=0 & \text { on } \partial \Omega
\end{aligned}\right.
$$

for suitable $s \in \mathbb{R}$ and $F \in \mathrm{L}^{q}(\Omega)^{3}, 1<q<\infty$. At first glance, it seems reasonable to regard (1.4) as a resolvent problem $(i s-A) v=F$ for a closed operator $A$ on the space of solenoidal vector fields in $\mathrm{L}^{q}(\Omega)^{3}$. However, the spectral analysis in this setting, which was carried out by Farwig and Neustupa [7,8], reveals that $i s, s \in \mathbb{R}$, belongs to the spectrum of $A$ when $s \in \omega \mathbb{Z}$, whereas well-posedness of the time-periodic problem requires invertibility of (1.4) for $s \in \omega \mathbb{Z}$. Therefore, we propose to investigate the problem in homogeneous Sobolev spaces instead. Although it is merely possible to derive the non-classical resolvent estimate (2.4) in this setting (see Theorem 2.1 below), we are nevertheless able to conclude a suitable solution theory for the linearization of (1.1). To this end, we shall employ a framework of functions with absolutely convergent Fourier series. Finally, a fixed-point argument yields the existence of a solution to the nonlinear problem (1.1) when the data $f, \xi$ and $\eta$ are "sufficiently small".

\section{Main Results}

In virtue of (1.2) we may assume $\lambda>0$ without loss of generality, and by (1.3) we have $\omega=2 \pi / \mathcal{T}>0$. To reformulate (1.1) in a non-dimensional way, we let the diameter $d>0$ of $\mathcal{B}$ serve as a characteristic length scale. We introduce the Reynolds number $\lambda^{\prime}:=\lambda \rho d / \mu$, the Taylor number $\omega^{\prime}:=\omega \rho d^{2} / \mu$, and the non-dimensional time and spatial variables $t^{\prime}=\omega t$ and $x^{\prime}=x / d$. In particular, $\Omega$ is transformed to $\Omega^{\prime}:=\{x / d \mid x \in \Omega\}$. We define $\alpha^{\prime}\left(t^{\prime}\right):=\alpha(t) \rho d / \mu$ and the non-dimensional functions

$$
u^{\prime}\left(t^{\prime}, x^{\prime}\right):=\frac{\rho d}{\mu} u(t, x), \quad \mathfrak{p}^{\prime}\left(t^{\prime}, x^{\prime}\right):=\frac{\rho d^{2}}{\mu^{2}} \mathfrak{p}(t, x), \quad f^{\prime}\left(t^{\prime}, x^{\prime}\right):=\frac{\rho d^{3}}{\mu^{2}} f(t, x),
$$

which are time-periodic with period $\mathcal{T}^{\prime}=2 \pi$ and can thus be identified with functions on the torus group $\mathbb{T}=\mathbb{R} / 2 \pi \mathbb{Z}$ with respect to time. Expressing (1.1) in these new quantities and omitting the primes, we obtain the non-dimensional formulation

$$
\left\{\begin{aligned}
\omega\left(\partial_{t} u+\mathrm{e}_{1} \wedge u-\mathrm{e}_{1} \wedge x \cdot \nabla u\right)-\alpha \partial_{1} u+u \cdot \nabla u=f+\Delta u-\nabla \mathfrak{p} & \text { in } \mathbb{T} \times \Omega, \\
\operatorname{div} u=0 & \text { in } \mathbb{T} \times \Omega, \\
u=\alpha \mathrm{e}_{1}+\omega \mathrm{e}_{1} \wedge x & \text { on } \mathbb{T} \times \partial \Omega, \\
\lim _{|x| \rightarrow \infty} u(t, x)=0 & \text { for } t \in \mathbb{T} .
\end{aligned}\right.
$$

Our analysis of (2.1) is based on the study of the linear time-periodic problem

$$
\left\{\begin{aligned}
\omega\left(\partial_{t} u+\mathrm{e}_{1} \wedge u-\mathrm{e}_{1} \wedge x \cdot \nabla u\right)-\Delta u-\lambda \partial_{1} u+\nabla \mathfrak{p}=f & \text { in } \mathbb{T} \times \Omega, \\
\operatorname{div} u=0 & \text { in } \mathbb{T} \times \Omega, \\
u=0 & \text { on } \mathbb{T} \times \partial \Omega,
\end{aligned}\right.
$$


and of the corresponding resolvent problem

$$
\left\{\begin{aligned}
\omega\left(i k v+\mathrm{e}_{1} \wedge v-\mathrm{e}_{1} \wedge x \cdot \nabla v\right)-\Delta v-\lambda \partial_{1} v+\nabla p=F & \text { in } \Omega, \\
\operatorname{div} v=0 & \text { in } \Omega, \\
v=0 & \text { on } \partial \Omega
\end{aligned}\right.
$$

for $k \in \mathbb{Z}$. For the latter we shall derive the following well-posedness result.

Theorem 2.1. Let $\Omega \subset \mathbb{R}^{3}$ be an exterior domain of class $\mathrm{C}^{3}$. Let $q \in(1,2), k \in \mathbb{Z}$ and $\lambda, \omega, \theta, B>0$ with $\lambda^{2} \leq \theta \omega \leq B$. For every $F \in \mathrm{L}^{q}(\Omega)^{3}$ there exists a solution $(v, p) \in \mathrm{W}_{\text {loc }}^{2, q}(\bar{\Omega})^{3} \times \mathrm{W}_{\text {loc }}^{1, q}(\bar{\Omega})$ to $(2.3)$ subject to the estimate

$$
\begin{array}{r}
\omega\left\|i k v+\mathrm{e}_{1} \wedge v-\mathrm{e}_{1} \wedge x \cdot \nabla v\right\|_{q}+\left\|\nabla^{2} v\right\|_{q}+\lambda\left\|\partial_{1} v\right\|_{q} \\
+\lambda^{1 / 2}\|v\|_{s_{1}}+\lambda^{1 / 4}\|\nabla v\|_{s_{2}}+\|\nabla p\|_{q} \leq C_{1}\|F\|_{q}
\end{array}
$$

for a constant $C_{1}=C_{1}(\Omega, q, \lambda, \omega)>0$ and $s_{1}=2 q /(2-q), s_{2}=4 q /(4-q)$. Additionally, if $(w, \mathfrak{q})$ is another solution to (2.3) in the function class defined by the norms on the left-hand side of (2.4), then $v=w$, and $p-\mathfrak{q}$ is a constant. Moreover, if $q \in\left(1, \frac{3}{2}\right)$, then the constant $C_{1}$ can be chosen independently of $\lambda$ and $\omega$ such that $C_{1}=C_{1}(\Omega, q, \theta, B)$.

Note that for $k=0$ we recover the well-known $\mathrm{L}^{q}$ theory for the corresponding stationary problem; see [13, Theorem VIII.8.1].

In order to transfer estimate (2.4) to the time-periodic setting without losing information on the dependencies of the constant $C_{1}$, we work within spaces $\mathrm{A}(\mathbb{T} ; X)$ of absolutely convergent $X$-valued Fourier series for suitable Banach spaces $X$; see (3.1) below. We establish the following solution theory for the time-periodic problem (2.2).

Theorem 2.2. Let $\Omega \subset \mathbb{R}^{3}$ be an exterior domain of class $\mathrm{C}^{3}$. Let $q \in(1,2)$ and $\lambda, \omega, \theta, B>0$ with $\lambda^{2} \leq \theta \omega \leq B$. For every $f \in \mathrm{A}\left(\mathbb{T} ; \mathrm{L}^{q}(\Omega)\right)^{3}$ there exists a solution $(u, \mathfrak{p})$ to $(2.2)$ subject to the estimate

$$
\begin{aligned}
& \omega\left\|\partial_{t} u+\mathrm{e}_{1} \wedge u-\mathrm{e}_{1} \wedge x \cdot \nabla u\right\|_{\mathrm{A}\left(\mathbb{T} ; \mathrm{L}^{q}(\Omega)\right)}+\left\|\nabla^{2} u\right\|_{\mathrm{A}\left(\mathbb{T} ; \mathrm{L}^{q}(\Omega)\right)}+\lambda\left\|\partial_{1} u\right\|_{\mathrm{A}\left(\mathbb{T} ; \mathrm{L}^{q}(\Omega)\right)} \\
& \quad+\lambda^{1 / 2}\|u\|_{\mathrm{A}\left(\mathbb{T} ; \mathrm{L}^{s_{1}}(\Omega)\right)}+\lambda^{1 / 4}\|\nabla u\|_{\mathrm{A}\left(\mathbb{T} ; \mathrm{L}^{s_{2}}(\Omega)\right)}+\|\nabla \mathfrak{p}\|_{\mathrm{A}\left(\mathbb{T} ; \mathrm{L}^{q}(\Omega)\right)} \\
& \quad \leq C_{1}\|f\|_{\mathrm{A}\left(\mathbb{T} ; \mathrm{L}^{q}(\Omega)\right)}
\end{aligned}
$$

for the constant $C_{1}$ from Theorem 2.1, and $s_{1}=2 q /(2-q), s_{2}=4 q /(4-q)$. Additionally, if $(w, \mathfrak{q})$ is another solution to (2.2) in the function class defined by the norms on the left-hand side of (2.5), then $u=w$ and $\mathfrak{p}=\mathfrak{q}+\mathfrak{q}_{0}$ for some (spatially constant) function $\mathfrak{q}_{0}: \mathbb{T} \rightarrow \mathbb{R}$.

In Sect. 6, we finally prove the following existence result on solutions to the nonlinear system (2.1).

Theorem 2.3. Let $\Omega \subset \mathbb{R}^{3}$ be an exterior domain of class $\mathrm{C}^{3}$, and let $q \in\left[\frac{12}{11}, \frac{4}{3}\right], \rho \in\left(\frac{3 q-3}{q}, 1\right)$ and $\theta>0$. Then there are constants $\kappa>0$ and $\lambda_{0}>0$ such that for all

$$
\lambda \in\left(0, \lambda_{0}\right), \quad \omega \in\left(\frac{\lambda^{2}}{\theta}, \infty\right)
$$

there exists $\varepsilon>0$ such that for all $f \in \mathrm{A}\left(\mathbb{T} ; \mathrm{L}^{q}(\Omega)\right)^{3}$ and $\alpha \in \mathrm{A}(\mathbb{T} ; \mathbb{R})$ with $\frac{\mathrm{d}}{\mathrm{d} t} \alpha \in \mathrm{A}(\mathbb{T} ; \mathbb{R})$ and

$$
\lambda=\frac{1}{2 \pi} \int_{0}^{2 \pi} \alpha(t) \mathrm{d} t, \quad \omega\left\|\frac{\mathrm{d}}{\mathrm{d} t} \alpha\right\|_{\mathrm{A}(\mathbb{T} ; \mathbb{R})} \leq \kappa \lambda^{\rho}, \quad\|\alpha-\lambda\|_{\mathrm{A}(\mathbb{T} ; \mathbb{R})}+\|f\|_{\mathrm{A}\left(\mathbb{T} ; L^{q}(\Omega)\right)} \leq \varepsilon
$$

there is a solution $(u, \mathfrak{p})$ to $(2.1)$ with

$$
\begin{gathered}
u \in \mathrm{A}\left(\mathbb{T} ; \mathrm{L}^{2 q /(2-q)}(\Omega)\right)^{3}, \quad \nabla u \in \mathrm{A}\left(\mathbb{T} ; \mathrm{L}^{4 q /(4-q)}(\Omega)\right)^{3 \times 3}, \\
\nabla^{2} u \in \mathrm{A}\left(\mathbb{T} ; \mathrm{L}^{q}(\Omega)\right)^{3 \times 3 \times 3}, \\
\partial_{t} u+\mathrm{e}_{1} \wedge u-\mathrm{e}_{1} \wedge x \cdot \nabla u, \partial_{1} u, \quad \nabla \mathfrak{p} \in \mathrm{A}\left(\mathbb{T} ; \mathrm{L}^{q}(\Omega)\right)^{3} .
\end{gathered}
$$


Remark 2.4. The lower bound $\frac{\lambda^{2}}{\theta} \leq \omega$ on the angular velocity in (2.6) may seem strange in light of the underlying physics of the problem since from a physical point of view, the limit $\omega \rightarrow 0$ towards the case of a non-rotating body seems uncritical. The lower bound on $\omega$ in (2.6) is an artifact of the change of coordinates into the rotating frame of reference employed in the mathematical analysis of the problem, which leads to a priori estimates with constants exhibiting a singular behavior as $\omega \rightarrow 0$. As a consequence, a lower bound on $\omega$ is required in Theorem 2.3 to obtain existence of a solution via a fixed-point iteration. A similar observation was made in the investigation of a steady flow past a rotating and translating obstacle carried out in [6]. Therefore, it is not surprising to see the same effect appearing in the more general time-periodic case investigated here.

\section{Preliminaries}

We use capital letters to denote global constants, while constants in small letters are local to the respective proof. When we want to emphasize that a constant $C$ depends on the quantities $\alpha, \beta, \gamma, \ldots$, we write $C(\alpha, \beta, \gamma, \ldots)$.

We denote points in $\mathbb{T} \times \mathbb{R}^{3}$ by $(t, x)$, where $t$ and $x=\left(x_{1}, x_{2}, x_{3}\right)$ are referred to as time and spatial variable. The symbol $\Omega$ always denotes an exterior domain, that is, $\Omega \subset \mathbb{R}^{3}$ is connected and the complement of a non-empty compact set. We always assume that the origin is not contained in $\Omega$.

Inner and outer product of two vectors $a, b \in \mathbb{R}^{3}$ are denoted by $a \cdot b$ and $a \wedge b$, respectively. For any radius $R>0$ we set $\mathrm{B}_{R}:=\left\{x \in \mathbb{R}^{3}|| x \mid<R\right\}, \mathrm{B}^{R}:=\left\{x \in \mathbb{R}^{3}|| x \mid>R\right\}$, and for a domain $D \subset \mathbb{R}^{3}$ we define $D_{R}:=D \cap \mathrm{B}_{R}$ and $D^{R}:=D \cap \mathrm{B}^{R}$.

For $q \in[1, \infty]$ and $k \in \mathbb{N}_{0}$, the symbols $\mathrm{L}^{q}(D)$ and $\mathrm{W}^{k, q}(D)$ denote usual Lebesgue and Sobolev spaces with associated norms $\|\cdot\|_{q}=\|\cdot\|_{q ; D}$ and $\|\cdot\|_{k, q}=\|\cdot\|_{k, q ; D}$, respectively. Furthermore, $\mathrm{W}_{0}^{1, q}(D)$ denotes the subset of functions in $\mathrm{W}^{1, q}(D)$ with vanishing boundary trace, and $\mathrm{W}^{-1, q}(D)$ (with norm $\|\cdot\|_{-1, q ; D}$ ) is the dual space of $\mathrm{W}_{0}^{1, q^{\prime}}(D)$ where $1 / q+1 / q^{\prime}=1$ with the usual convention $1 / \infty:=0$. Moreover, $\mathrm{L}_{\sigma}^{2}(D)$ denotes the set of solenoidal vector fields in $\mathrm{L}^{2}(D)^{3}$, that is,

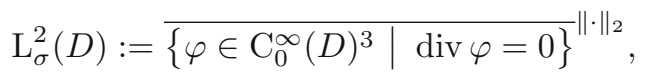

and $\mathcal{P}_{\mathrm{H}}$ is the corresponding Helmholtz projection that maps $\mathrm{L}^{2}(D)^{3}$ onto $\mathrm{L}_{\sigma}^{2}(D)$.

We always identify $2 \pi$-periodic functions with functions on the torus group $\mathbb{T}:=\mathbb{R} / 2 \pi \mathbb{Z}$, which is usually represented by the set $[0,2 \pi)$. We consider $\mathbb{T}$ and $G:=\mathbb{T} \times \mathbb{R}^{3}$ as locally compact abelian groups. The (normalized) Haar measure on $\mathbb{T}$ is given by

$$
\forall f \in \mathrm{C}(\mathbb{T}): \quad \int_{\mathbb{T}} f \mathrm{~d} t:=\frac{1}{2 \pi} \int_{0}^{2 \pi} f(t) \mathrm{d} t,
$$

and $G$ is equipped with the corresponding product measure. Recall that the dual group of $\mathbb{T}$ can be identified with $\widehat{\mathbb{T}}=\mathbb{Z}$ and that of $G$ with $\widehat{G}:=\mathbb{Z} \times \mathbb{R}^{3}$.

For $H=\mathbb{T}$ or $H=G$, the space $\mathscr{S}(H)$ is the Schwartz-Bruhat space of generalized Schwartz functions on $H$, and $\mathscr{S}^{\prime}(H)$ denotes the corresponding dual space of tempered distributions; see $[1,4]$ for precise definitions. The Fourier transform on $\mathbb{T}$ and $G$ and the respective inverses are given by

$$
\begin{array}{lr}
\mathscr{F}_{\mathbb{T}}: \mathscr{S}(\mathbb{T}) \rightarrow \mathscr{S}(\mathbb{Z}), & \mathscr{F}_{\mathbb{T}}[u](k):=\int_{\mathbb{T}} u(t) \mathrm{e}^{-i k t} \mathrm{~d} t, \\
\mathscr{F}_{\mathbb{T}}^{-1}: \mathscr{S}(\mathbb{Z}) \rightarrow \mathscr{S}(\mathbb{T}), & \mathscr{F}_{\mathbb{T}}^{-1}[w](t):=\sum_{k \in \mathbb{Z}} w(k) \mathrm{e}^{i k t},
\end{array}
$$

and

$$
\mathscr{F}_{G}: \mathscr{S}(G) \rightarrow \mathscr{S}(\widehat{G}), \quad \quad \mathscr{F}_{G}[u](k, \xi):=\int_{\mathbb{T}} \int_{\mathbb{R}^{n}} u(t, x) \mathrm{e}^{-i x \cdot \xi-i k t} \mathrm{~d} x \mathrm{~d} t,
$$




$$
\mathscr{F}_{G}^{-1}: \mathscr{S}(\widehat{G}) \rightarrow \mathscr{S}(G), \quad \mathscr{F}_{G}^{-1}[w](t, x):=\sum_{k \in \mathbb{Z}} \int_{\mathbb{R}^{n}} w(k, \xi) \mathrm{e}^{i x \cdot \xi+i k t} \mathrm{~d} \xi,
$$

provided the Lebesgue measure d $\xi$ is correctly normalized. By duality, $\mathscr{F}_{\mathbb{T}}$ and $\mathscr{F}_{G}$ extend to homeomorphisms $\mathscr{F}_{\mathbb{T}}: \mathscr{S}^{\prime}(\mathbb{T}) \rightarrow \mathscr{S}^{\prime}(\mathbb{Z})$ and $\mathscr{F}_{G}: \mathscr{S}^{\prime}(G) \rightarrow \mathscr{S}^{\prime}(\widehat{G})$, respectively.

Furthermore, we introduce the Sobolev space

$$
\mathrm{W}^{1,2, q}(\mathbb{T} \times D):={\overline{\mathrm{C}_{0}^{\infty}(\mathbb{T} \times \bar{D})}}^{\|\cdot\|_{1,2, q}}, \quad\|f\|_{1,2, q}:=\left(\left\|\partial_{t} f\right\|_{q}^{q}+\sum_{k=0}^{2}\left\|\nabla^{k} f\right\|_{q}^{q}\right)^{\frac{1}{q}},
$$

where $\mathrm{C}_{0}^{\infty}(\mathbb{T} \times \bar{D})$ denotes the space of smooth functions of compact support on $\mathbb{T} \times \bar{D}$.

Let $X$ be a Banach space. We introduce the projections $\mathcal{P}$ and $\mathcal{P}_{\perp}$ by

$$
\mathcal{P} u:=\int_{\mathbb{T}} u(t) \mathrm{d} t, \quad \mathcal{P}_{\perp}:=\mathrm{Id}-\mathcal{P}
$$

for $u \in \mathrm{L}^{1}(\mathbb{T} ; X)$. Note that $\mathcal{P} u \in X$ is time-independent, and we have the decomposition $u=\mathcal{P} u+\mathcal{P}_{\perp} u$ into the steady-state part $\mathcal{P} u$ and the purely periodic part $\mathcal{P}_{\perp} u$ of $u$.

Our analysis of the time-periodic problems (2.1) and (2.2) will be carried out within spaces of functions with absolutely convergent Fourier series defined by

$$
\begin{aligned}
\mathrm{A}(\mathbb{T} ; X) & :=\left\{f: \mathbb{T} \rightarrow X \mid f(t)=\sum_{k \in \mathbb{Z}} f_{k} \mathrm{e}^{i k t}, f_{k} \in X, \sum_{k \in \mathbb{Z}}\left\|f_{k}\right\|_{X}<\infty\right\}, \\
\|f\|_{\mathrm{A}(\mathbb{T} ; X)} & :=\sum_{k \in \mathbb{Z}}\left\|f_{k}\right\|_{X} .
\end{aligned}
$$

Observe that $\mathrm{A}(\mathbb{T} ; X)$ is the Banach space that coincides with $\mathscr{F}_{\mathbb{T}}^{-1}\left[\ell^{1}(\mathbb{Z} ; X)\right]$, which embeds into the $X$-valued continuous functions on $\mathbb{T}$. It is well known that the scalar-valued space $\mathrm{A}(\mathbb{T} ; \mathbb{R})$ is an algebra with respect to pointwise multiplication, the so-called Wiener algebra. One can exploit this property to derive estimates in the $X$-valued case. For example, one readily shows the following correspondences of Hölder's inequality and interpolation inequalities.

Proposition 3.1. Let $D \subset \mathbb{R}^{n}, n \in \mathbb{N}$, be an open set and $p, q, r \in[1, \infty]$ such that $1 / p+1 / q=1 / r$. Moreover, let $f \in \mathrm{A}\left(\mathbb{T} ; \mathrm{L}^{p}(D)\right)$ and $g \in \mathrm{A}\left(\mathbb{T} ; \mathrm{L}^{q}(D)\right)$. Then $f g \in \mathrm{A}\left(\mathbb{T} ; \mathrm{L}^{r}(D)\right)$ and

$$
\|f g\|_{\mathrm{A}\left(\mathbb{T} ; \mathrm{L}^{r}(D)\right)} \leq\|f\|_{\mathrm{A}\left(\mathbb{T} ; \mathrm{L}^{p}(D)\right)}\|g\|_{\mathrm{A}\left(\mathbb{T} ; \mathrm{L}^{q}(D)\right)} .
$$

Proof. By assumption we have $f=\mathscr{F}_{\mathbb{T}}^{-1}\left[\left(f_{k}\right)\right]$ and $g=\mathscr{F}_{\mathbb{T}}^{-1}\left[\left(g_{k}\right)\right]$ for elements $\left(f_{k}\right) \in \ell^{1}\left(\mathbb{Z} ; \mathrm{L}^{p}(D)\right)$ and $\left(g_{k}\right) \in \ell^{1}\left(\mathbb{Z} ; \mathrm{L}^{q}(D)\right)$. Then $f g=\mathscr{F}_{\mathbb{T}}^{-1}\left[\left(f_{k}\right) *_{\mathbb{Z}}\left(g_{k}\right)\right]$ and

$$
\begin{aligned}
\|f g\|_{\mathrm{A}\left(\mathbb{T} ; \mathrm{L}^{r}(D)\right)} & =\sum_{k \in \mathbb{Z}}\left\|\sum_{\ell \in \mathbb{Z}} f_{\ell} g_{k-\ell}\right\|_{\mathrm{L}^{r}(D)} \leq \sum_{k \in \mathbb{Z}} \sum_{\ell \in \mathbb{Z}}\left\|f_{\ell} g_{k-\ell}\right\|_{\mathrm{L}^{r}(D)} \\
& \leq \sum_{k \in \mathbb{Z}} \sum_{\ell \in \mathbb{Z}}\left\|f_{\ell}\right\|_{\mathrm{L}^{p}(D)}\left\|g_{k-\ell}\right\|_{\mathrm{L}^{q}(D)}=\|f\|_{\mathrm{A}\left(\mathbb{T} ; \mathrm{L}^{p}(D)\right)}\|g\|_{\mathrm{A}\left(\mathbb{T} ; \mathrm{L}^{q}(D)\right)},
\end{aligned}
$$

where the last estimate is due to Hölder's inequality.

Proposition 3.2. Let $D \subset \mathbb{R}^{n}, n \in \mathbb{N}$, be an open set and $p, q, r \in[1, \infty]$ such that $(1-\theta) / p+\theta / q=1 / r$ for some $\theta \in[0,1]$, and let $f \in \mathrm{A}\left(\mathbb{T} ; \mathrm{L}^{p}(D)\right) \cap \mathrm{A}\left(\mathbb{T} ; \mathrm{L}^{q}(D)\right)$. Then $f \in \mathrm{A}\left(\mathbb{T} ; \mathrm{L}^{r}(D)\right)$ and

$$
\|f\|_{\mathrm{A}\left(\mathbb{T} ; \mathrm{L}^{r}(D)\right)} \leq\|f\|_{\mathrm{A}\left(\mathbb{T} ; \mathrm{L}^{p}(D)\right)}^{1-\theta}\|f\|_{\mathrm{A}\left(\mathbb{T} ; \mathrm{L}^{q}(D)\right)}^{\theta} .
$$

Proof. We have $f=\mathscr{F}_{\mathbb{T}}^{-1}\left[\left(f_{k}\right)\right]$ for an element $\left(f_{k}\right) \in \ell^{1}\left(\mathbb{Z} ; \mathrm{L}^{p}(D) \cap \mathrm{L}^{q}(D)\right)$. The classical interpolation inequality for Lebesgue spaces yields

$$
\begin{aligned}
\|f\|_{\mathrm{A}\left(\mathbb{T} ; \mathrm{L}^{r}(D)\right)}=\sum_{k \in \mathbb{Z}}\left\|f_{k}\right\|_{\mathrm{L}^{r}(D)} & \leq \sum_{k \in \mathbb{Z}}\left\|f_{k}\right\|_{\mathrm{L}^{p}(D)}^{1-\theta}\left\|f_{k}\right\|_{\mathrm{L}^{q}(D)}^{\theta} \\
& \leq\|f\|_{\mathrm{A}\left(\mathbb{T} ; \mathrm{L}^{p}(D)\right)}^{1-\theta}\|f\|_{\mathrm{A}\left(\mathbb{T} ; \mathrm{L}^{q}(D)\right)}^{\theta}
\end{aligned}
$$


where the last estimate follows from Hölder's inequality on $\mathbb{Z}$.

\section{Embedding Theorem}

This section deals with embedding properties of Sobolev spaces of time-periodic functions. The embedding theorem below is a refinement of [18, Theorem 4.1] adapted to the time-scaling employed in (2.1). Clearly, embeddings of the steady-state part $\mathcal{P} u$ are independent of the actual period. Therefore, we only consider the case of purely periodic functions. For the sake of generality, we establish the following theorem in arbitrary dimension $n \geq 2$.

Theorem 4.1. Let $n \geq 2, \omega>0$ and $q \in(1, \infty)$. For $\alpha \in[0,2]$ with $\alpha q<2$ and $(2-\alpha) q<n$ let

$$
r_{0}:=\frac{2 q}{2-\alpha q}, \quad p_{0}:=\frac{n q}{n-(2-\alpha) q},
$$

and for $\beta \in[0,1]$ with $\beta q<2$ and $(1-\beta) q<n$ let

$$
r_{1}:=\frac{2 q}{2-\beta q}, \quad p_{1}:=\frac{n q}{n-(1-\beta) q} .
$$

Then the inequality

$$
\omega^{\alpha / 2}\|u\|_{\mathrm{L}^{r_{0}\left(\mathbb{T} ; \mathrm{L}^{p_{0}}\left(\mathbb{R}^{n}\right)\right)}}+\omega^{\beta / 2}\|\nabla u\|_{\mathrm{L}^{r_{1}\left(\mathbb{T} ; \mathrm{L}^{p_{1}}\left(\mathbb{R}^{n}\right)\right)}} \leq C_{2}\left(\omega\left\|\partial_{t} u\right\|_{q}+\left\|\nabla^{2} u\right\|_{q}\right)
$$

holds for all $u \in \mathcal{P}_{\perp} \mathrm{W}^{1,2, q}\left(\mathbb{T} \times \mathbb{R}^{n}\right)$ and a constant $C_{2}=C_{2}(n, q, \alpha, \beta)>0$.

Proof. Since the proof is analogue to [18, Proof of Theorem 4.1], we merely give a brief sketch here. Without restriction we may assume $u \in \mathscr{S}(G)$. Due to the assumption $u=\mathcal{P}_{\perp} u$, we have $\mathscr{F}_{G}[u]=$ $\left(1-\delta_{\mathbb{Z}}\right) \mathscr{F}_{G}[u]$, where $\delta_{\mathbb{Z}}$ is the delta distribution on $\mathbb{Z}$. Utilizing the Fourier transform, we thus derive the identity

$$
\begin{aligned}
u & =\mathscr{F}_{G}^{-1}\left[\frac{1-\delta_{\mathbb{Z}}(k)}{|\xi|^{2}+i \omega k} \mathscr{F}_{G}\left[\omega \partial_{t} u-\Delta u\right]\right] \\
& =\omega^{-\alpha / 2} \mathscr{F}_{\mathbb{R}^{n}}^{-1}\left[|\xi|^{\alpha-2}\right] *_{\mathbb{R}^{n}} \mathscr{F}_{\mathbb{T}}^{-1}\left[\left(1-\delta_{\mathbb{Z}}\right)|k|^{-\alpha / 2}\right] *_{\mathbb{T}} F,
\end{aligned}
$$

where

$$
F:=\mathscr{F}_{G}^{-1}\left[M_{\omega}(k, \xi) \mathscr{F}_{G}\left[\omega \partial_{t} u-\Delta u\right]\right], \quad M_{\omega}(k, \xi):=\frac{|\omega k|^{\alpha / 2}|\xi|^{2-\alpha}\left(1-\delta_{\mathbb{Z}}(k)\right)}{|\xi|^{2}+i \omega k} .
$$

Employing the so-called transference principle for Fourier multipliers (see $[3,4]$ ) together with the Marcinkiewicz multiplier theorem, one readily verifies that $M_{\omega}$ is an $\mathrm{L}^{q}(G)$ multiplier for any $q \in(1, \infty)$ such that

$$
\|F\|_{q} \leq c_{0}\left\|\omega \partial_{t} u-\Delta u\right\|_{q} \leq c_{0}\left(\omega\left\|\partial_{t} u\right\|_{q}+\left\|\nabla^{2} u\right\|_{q}\right)
$$

with $c_{0}$ independent of $\omega$. Moreover, when we choose $[-\pi, \pi)$ as a realization of $\mathbb{T}$, we obtain

$$
\gamma_{\alpha}(t):=\mathscr{F}_{\mathbb{T}}^{-1}\left[\left(1-\delta_{\mathbb{Z}}\right)|k|^{-\alpha / 2}\right](t)=c_{1} t^{-1+\alpha / 2}+h(t),
$$

for some $h \in \mathrm{C}^{\infty}(\mathbb{T})$; see for example [24, Example 3.1.19]. In particular, this yields $\gamma_{\alpha} \in \mathrm{L}^{\frac{1}{1-\alpha / 2}, \infty}(\mathbb{T})$, so that Young's inequality implies that the mapping $\varphi \mapsto \gamma_{\alpha} * \varphi$ extends to a bounded operator $\mathrm{L}^{q}(\mathbb{T}) \rightarrow$ $\mathrm{L}^{r_{0}}(\mathbb{T})$. Moreover, it is well known that the mapping $\varphi \mapsto \mathscr{F}_{\mathbb{R}^{n}}^{-1}\left[|\xi|^{\alpha-2}\right] * \varphi$ extends to a bounded operator $\mathrm{L}^{q}\left(\mathbb{R}^{n}\right) \rightarrow \mathrm{L}^{p_{0}}\left(\mathbb{R}^{n}\right)$; see [25, Theorem 6.1.13]. Recalling (4.2), we thus have

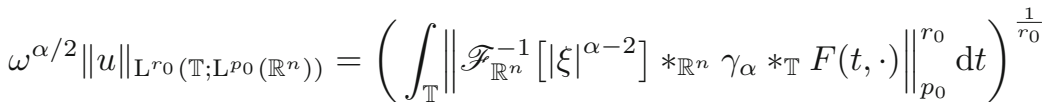

$$
\begin{aligned}
& \leq c_{2}\left(\int_{\mathbb{T}}\left\|\gamma_{\alpha} * \mathbb{T} F(t, \cdot)\right\|_{q}^{r_{0}} \mathrm{~d} t\right)^{\frac{1}{r_{0}}} \leq c_{3}\left(\int_{\mathbb{R}^{n}}\left\|\gamma_{\alpha} *_{\mathbb{T}} F(\cdot, x)\right\|_{r_{0}}^{q} \mathrm{~d} x\right)^{\frac{1}{q}}
\end{aligned}
$$




$$
\leq c_{4}\|F\|_{q} \leq c_{5}\left(\omega\left\|\partial_{t} u\right\|_{q}+\left\|\nabla^{2} u\right\|_{q}\right),
$$

where Minkowski's integral inequality is used in the second estimate. This is the asserted inequality for $u$. The estimate of $\nabla u$ follows in the same way.

Remark 4.2. Note that the term on the right-hand side of (4.1) defines a norm equivalent to $\|\cdot\|_{1,2, q}$ on $\mathcal{P}_{\perp} \mathrm{W}^{1,2, q}(\mathbb{T} \times \Omega)$ due to Poincaré's inequality on $\mathbb{T}$.

Remark 4.3. Theorem 4.1 can be generalized to the setting of an exterior domain $\Omega \subset \mathbb{R}^{n}$ by means of Sobolev extensions. However, to maintain estimate (4.1), one has to construct a specific extension operator that respects the homogeneous second-order Sobolev norm. To this end, one can make use of results from [2].

\section{Linear Theory}

This section is dedicated to the investigation of the resolvent problem (2.3) and the linear time-periodic problem (2.2). After having shown Theorem 2.1, we establish Theorem 2.2 as an immediate consequence hereof.

\subsection{The Whole Space}

To study the problems (2.2) and (2.3) in an exterior domain, we first consider the case $\Omega=\mathbb{R}^{3}$. In this whole-space setting one can namely change coordinates back to the non-rotating inertial frame and thereby reduce the study of (2.2) to an investigation of the time-periodic Oseen problem without rotation terms, which was analyzed in $[18,32]$. In this section, we set

$$
s_{1}:=\frac{2 q}{2-q}, \quad s_{2}:=\frac{4 q}{4-q}, \quad s_{3}:=\frac{8 q}{8-q} .
$$

for appropriately fixed $q$.

Theorem 5.1. Let $q \in(1,2)$ and $\lambda, \omega, \theta>0$ with $\lambda^{2} \leq \theta \omega$. For every $f \in \mathrm{L}^{q}\left(\mathbb{T} \times \mathbb{R}^{3}\right)^{3}$ there exists a solution $(u, \mathfrak{p}) \in \mathscr{S}^{\prime}\left(\mathbb{T} \times \mathbb{R}^{3}\right)^{3+1}$ to

$$
\left\{\begin{aligned}
\omega \partial_{t} u-\Delta u-\lambda \partial_{1} u+\nabla \mathfrak{p}=f & & \text { in } \mathbb{T} \times \mathbb{R}^{3}, \\
\operatorname{div} u=0 & & \text { in } \mathbb{T} \times \mathbb{R}^{3},
\end{aligned}\right.
$$

with $\partial_{t} u, \nabla^{2} u, \nabla \mathfrak{p} \in \mathrm{L}^{q}\left(\mathbb{T} \times \mathbb{R}^{3}\right)$. Moreover, there exist constants $C_{3}=C_{3}(q)>0$ and $C_{4}=C_{4}(q, \theta)>0$ such that

$$
\begin{aligned}
& \left\|\nabla^{2} \mathcal{P} u\right\|_{q}+\lambda\left\|\partial_{1} \mathcal{P} u\right\|_{q}+\lambda^{1 / 2}\|\mathcal{P} u\|_{s_{1}}+\lambda^{1 / 4}\|\nabla \mathcal{P} u\|_{s_{2}}+\|\nabla \mathcal{P} \mathfrak{p}\|_{q} \leq C_{3}\|\mathcal{P} f\|_{q} \\
& \omega\left\|\partial_{t} \mathcal{P}_{\perp} u\right\|_{q}+\left\|\nabla^{2} \mathcal{P}_{\perp} u\right\|_{q}+\lambda\left\|\partial_{1} \mathcal{P}_{\perp} u\right\|_{q}+\|\nabla \mathcal{P} \perp \mathfrak{p}\|_{q} \leq C_{4}\left\|\mathcal{P}_{\perp} f\right\|_{q} .
\end{aligned}
$$

Additionally, if $(w, \mathfrak{q}) \in \mathscr{S}^{\prime}\left(\mathbb{T} \times \mathbb{R}^{3}\right)^{3+1}$ is another solution to $(5.1)$, then $\mathcal{P}_{\perp} u=\mathcal{P}_{\perp} w$, and $\mathcal{P} u-\mathcal{P} w$ is a polynomial in each component, and $\mathfrak{p}-\mathfrak{q}=\mathfrak{p}_{0}$, where $\mathfrak{p}_{0}(t, \cdot)$ is a polynomial for each $t \in \mathbb{T}$.

Proof. We decompose (5.1) into two problems by splitting $u=\mathcal{P} u+\mathcal{P}_{\perp} u=: u_{\mathrm{s}}+u_{\mathrm{p}}$ and $\mathfrak{p}=\mathcal{P} \mathfrak{p}+\mathcal{P}_{\perp} \mathfrak{p}=$ : $\mathfrak{p}_{\mathrm{s}}+\mathfrak{p}_{\mathrm{p}}$. For the steady-state part $\left(u_{\mathrm{s}}, \mathfrak{p}_{\mathrm{s}}\right)$ we obtain the system

$$
\left\{\begin{aligned}
-\Delta u_{\mathrm{s}}-\lambda \partial_{1} u_{\mathrm{s}}+\nabla \mathfrak{p}_{\mathrm{s}} & =\mathcal{P} f & & \text { in } \mathbb{R}^{3}, \\
\operatorname{div} u_{\mathrm{s}} & =0 & & \text { in } \mathbb{R}^{3},
\end{aligned}\right.
$$


which is the classical steady-state Oseen problem. The existence of a time-independent solution $\left(u_{\mathrm{s}}, \mathfrak{p}_{\mathrm{s}}\right)$ satisfying estimate (5.2) is well known; see for example [13, Theorem VII.4.1]. The remaining purely periodic part $\left(u_{\mathrm{p}}, \mathfrak{p}_{\mathrm{p}}\right)$ must solve (5.1), but with purely periodic right-hand side $\mathcal{P}_{\perp} f$. We define

$$
\begin{aligned}
U(t, x) & :=u_{\mathrm{p}}\left(t, \omega^{-1 / 2} x\right), \\
\mathfrak{P}(t, x) & :=\omega^{-1 / 2} \mathfrak{p}_{\mathrm{p}}\left(t, \omega^{-1 / 2} x\right), \\
F(t, x) & :=\omega^{-1} \mathcal{P}_{\perp} f\left(t, \omega^{-1 / 2} x\right),
\end{aligned}
$$

which leads to the system

$$
\left\{\begin{array}{rlrl}
\partial_{t} U-\Delta U-\tilde{\lambda} \partial_{1} U+\nabla \mathfrak{P}=F & & \text { in } \mathbb{T} \times \mathbb{R}^{3}, \\
\operatorname{div} U=0 & \text { in } \mathbb{T} \times \mathbb{R}^{3},
\end{array}\right.
$$

where $\tilde{\lambda}=\lambda \omega^{-1 / 2}$. From [32, Theorem 2.1] we conclude the existence of a unique solution $(U, \mathfrak{P})$ that satisfies the estimate

$$
\|U\|_{1,2, q}+\|\nabla \mathfrak{P}\|_{q} \leq c_{0}\|F\|_{q},
$$

where $c_{0}$ is a polynomial in $\tilde{\lambda}$ and can thus be bounded uniformly in $\tilde{\lambda} \in(0, \sqrt{\theta}]$. Estimate (5.3) with the asserted dependency of the constant $C_{4}$ follows after reversing the applied scaling.

The uniqueness statement is readily shown by means of the Fourier transform on $G=\mathbb{T} \times \mathbb{R}^{3}$. We consider (5.1) with $f=0$ and apply the divergence operator to (5.1) 1 . This yields $\Delta \mathfrak{p}=0$ and thus $|\xi|^{2} \mathscr{F}_{\mathbb{R}^{3}}[\mathfrak{p}(t, \cdot)]=0$ for all $t \in \mathbb{T}$. Therefore, we obtain supp $\mathscr{F}_{\mathbb{R}^{3}}[\mathfrak{p}(t, \cdot)] \subset\{0\}$, so that $\mathfrak{p}(t, \cdot)$ is a polynomial for all $t \in \mathbb{T}$. Next we apply the Fourier transform to $(5.1)_{1}$ to deduce $\left(i \omega k+|\xi|^{2}-i \xi_{1}\right) \mathscr{F}_{G}[u]+i \xi \mathscr{F}_{G}[\mathfrak{p}]=0$. Multiplying with the symbol of the Helmholtz projection $\mathrm{I}-\xi \otimes \xi /|\xi|^{2}$ and utilizing $\operatorname{div} u=0$, we obtain $\left(i \omega k+|\xi|^{2}-i \xi_{1}\right) \mathscr{F}_{G}[u]=0$, which yields supp $\mathscr{F}_{G}[u] \subset\{(0,0)\}$. Since $\mathcal{P}_{\perp} u=\mathscr{F}_{G}^{-1}\left[\left(1-\delta_{\mathbb{Z}}\right) \mathscr{F}_{G}[u]\right]$, it follows that $\mathcal{P}_{\perp} u=0$, and that each component of $\mathcal{P} u$ is a polynomial. This completes the proof.

Remark 5.2. In the setting of Theorem 5.1 we can write the estimate for the steady-state part $\left(u_{\mathrm{s}}, \mathfrak{p}_{\mathrm{s}}\right)=$ $(\mathcal{P} u, \mathcal{P} \mathfrak{p})$ and the purely periodic part $\left(u_{\mathrm{p}}, \mathfrak{p}_{\mathrm{p}}\right)=\left(\mathcal{P}_{\perp} u, \mathcal{P}_{\perp} \mathfrak{p}\right)$ in a more condensed way: From the embeddings established in Theorem 4.1 we deduce

$$
\begin{aligned}
& \omega^{1 / 4}\left\|u_{\mathrm{p}}\right\|_{\mathrm{L}^{s_{2}}\left(\mathbb{T} ; \mathrm{L}^{s_{1}}\left(\mathbb{R}^{3}\right)\right)}+\omega^{1 / 8}\left\|\nabla u_{\mathrm{p}}\right\|_{\mathrm{L}^{s_{3}}\left(\mathbb{T} ; \mathrm{L}^{s_{2}}\left(\mathbb{R}^{3}\right)\right)} \\
& \quad \leq C_{5}\left(\omega\left\|\partial_{t} u_{\mathrm{p}}\right\|_{\mathrm{L}^{q}\left(\mathbb{T} \times \mathbb{R}^{3}\right)}+\left\|u_{\mathrm{p}}\right\|_{\mathrm{L}^{q}\left(\mathbb{T} \times \mathbb{R}^{3}\right)}\right) .
\end{aligned}
$$

Recalling Remark 4.2, we see that (5.2) and (5.3) can be formulated as

$$
\begin{aligned}
\omega\left\|\partial_{t} u\right\|_{q} & +\left\|\nabla^{2} u\right\|_{q}+\lambda\left\|\partial_{1} u\right\|_{q}+\lambda^{1 / 2}\|u\|_{L^{s_{2}}\left(\mathbb{T} ; L^{s_{1}}\left(\mathbb{R}^{3}\right)\right)} \\
& +\lambda^{1 / 4}\|\nabla u\|_{L^{s_{3}}\left(\mathbb{T} ; L^{s_{2}}\left(\mathbb{R}^{3}\right)\right)}+\|\nabla \mathfrak{p}\|_{q} \leq C_{6}\|f\|_{q}
\end{aligned}
$$

for a constant $C_{6}=C_{6}(q, \theta)$ as long as $\lambda^{2} \leq \theta \omega$

With Theorem 5.1 we now solve the linear problem (2.2) for $\Omega=\mathbb{R}^{3}$ and $f \in \mathrm{L}^{q}\left(\mathbb{T} \times \mathbb{R}^{3}\right)^{3}$.

Theorem 5.3. Let $q \in(1,2)$ and $\lambda, \omega, \theta>0$ with $\lambda^{2} \leq \theta \omega$. For every $f \in \mathrm{L}^{q}\left(\mathbb{T} \times \mathbb{R}^{3}\right)^{3}$ there exists a solution $(u, \mathfrak{p}) \in \mathscr{S}^{\prime}\left(\mathbb{T} \times \mathbb{R}^{3}\right)^{3+1}$ to

$$
\left\{\begin{aligned}
\omega\left(\partial_{t} u+\mathrm{e}_{1} \wedge u-\mathrm{e}_{1} \wedge x \cdot \nabla u\right)-\Delta u-\lambda \partial_{1} u+\nabla \mathfrak{p}=f & \text { in } \mathbb{T} \times \mathbb{R}^{3}, \\
\operatorname{div} u=0 & \text { in } \mathbb{T} \times \mathbb{R}^{3},
\end{aligned}\right.
$$

with $\nabla^{2} u, \partial_{1} u, \nabla \mathfrak{p} \in \mathrm{L}^{q}\left(\mathbb{T} \times \mathbb{R}^{3}\right)$. Moreover, there exists a constant $C_{7}=C_{7}(q, \theta)>0$ such that

$$
\begin{aligned}
& \omega\left\|\partial_{t} u+\mathrm{e}_{1} \wedge u-\mathrm{e}_{1} \wedge x \cdot \nabla u\right\|_{\mathrm{L}^{q}\left(\mathbb{T} \times \mathbb{R}^{3}\right)}+\left\|\nabla^{2} u\right\|_{\mathrm{L}^{q}\left(\mathbb{T} \times \mathbb{R}^{3}\right)}+\lambda\left\|\partial_{1} u\right\|_{\mathrm{L}^{q}\left(\mathbb{T} \times \mathbb{R}^{3}\right)} \\
& +\lambda^{1 / 2}\|u\|_{L^{s_{2}}\left(\mathbb{T} ; L^{s_{1}}\left(\mathbb{R}^{3}\right)\right)}+\lambda^{1 / 4}\|\nabla u\|_{L^{s_{3}}\left(\mathbb{T} ; L^{s_{2}}\left(\mathbb{R}^{3}\right)\right)}+\|\nabla \mathfrak{p}\|_{\mathrm{L}^{q}\left(\mathbb{T} \times \mathbb{R}^{3}\right)} \\
& \leq C_{7}\|f\|_{\mathrm{L}^{q}\left(\mathbb{T} \times \mathbb{R}^{3}\right)} \text {. }
\end{aligned}
$$


Additionally, if $(w, \mathfrak{q}) \in \mathscr{S}^{\prime}\left(\mathbb{T} \times \mathbb{R}^{3}\right)^{3+1}$ is another solution to (5.5) with $w \in \mathrm{L}^{r}\left(\mathbb{T} \times \mathbb{R}^{3}\right)$ for some $r \in[1, \infty)$, then $u=w$, and $\mathfrak{p}-\mathfrak{q}=\mathfrak{q}_{0}$ for some spatially constant function $\mathfrak{q}_{0}: \mathbb{T} \rightarrow \mathbb{R}$.

Proof. Let

$$
Q(t):=\left(\begin{array}{ccc}
1 & 0 & 0 \\
0 & \cos (t) & -\sin (t) \\
0 & \sin (t) & \cos (t)
\end{array}\right)
$$

be the matrix corresponding to the rotation with angular velocity $\mathrm{e}_{1}$. Define

$$
\begin{aligned}
U(t, y) & :=Q(t) u\left(t, Q(t)^{\top} y\right), \\
\mathfrak{P}(t, y) & :=\mathfrak{p}\left(t, Q(t)^{\top} y\right), \\
F(t, y) & :=Q(t) f\left(t, Q(t)^{\top} y\right)
\end{aligned}
$$

with the new spatial variable $y=Q(t) x$. Due to

$$
\partial_{t} U(t, y)=Q(t)\left(\partial_{t} u(t, x)+\mathrm{e}_{1} \wedge u(t, x)-\mathrm{e}_{1} \wedge x \cdot \nabla u(t, x)\right),
$$

the functions $u, \mathfrak{p}$ and $f$ satisfy (5.5) if and only if

$$
\left\{\begin{aligned}
\omega \partial_{t} U-\Delta U-\lambda \partial_{1} U+\nabla \mathfrak{P}=F & \text { in } \mathbb{T} \times \mathbb{R}^{3}, \\
\operatorname{div} U=0 & \text { in } \mathbb{T} \times \mathbb{R}^{3} .
\end{aligned}\right.
$$

The assertions in Theorem 5.3 are now a direct consequence of Theorem 5.1 and estimate (5.4).

Remark 5.4. As for the corresponding steady-state problem (see for example [13, Theorem VIII.8.1]), one can extend Theorem 5.3 to the case of an exterior domain $\Omega$ for $f \in \mathrm{L}^{q}(\mathbb{T} \times \Omega)$, but it is not clear to the authors whether or not the constant in the resulting a priori estimate can then be chosen independently of $\lambda$ and $\omega$. Observe that such an independence is obtained in the functional setting of Theorem 2.2 where $f \in \mathrm{A}\left(\mathbb{T} ; \mathrm{L}^{q}(\Omega)\right)$. Since we solve the nonlinear problem (2.1) via a fixed-point iteration which requires $\lambda$ and $\omega$ to be chosen sufficiently small, it crucial to obtain an estimate with the constant independent of $\lambda$ and $\omega$.

From Theorem 5.3 we can extract a similar result for the resolvent problem (2.3) in the whole space.

Theorem 5.5. Let $q \in(1,2), k \in \mathbb{Z}$ and $\lambda, \omega, \theta>0$ with $\lambda^{2} \leq \theta \omega$. For every $F \in \mathrm{L}^{q}\left(\mathbb{R}^{3}\right)^{3}$ there exists a solution $(v, p) \in \mathscr{S}^{\prime}\left(\mathbb{R}^{3}\right)^{3+1}$ to

$$
\left\{\begin{aligned}
\omega\left(i k v+\mathrm{e}_{1} \wedge v-\mathrm{e}_{1} \wedge x \cdot \nabla v\right)-\Delta v-\lambda \partial_{1} v+\nabla p=F & \text { in } \mathbb{R}^{3}, \\
\operatorname{div} v=0 & \text { in } \mathbb{R}^{3},
\end{aligned}\right.
$$

and a constant $C_{8}=C_{8}(q, \theta)>0$ with

$$
\begin{aligned}
\omega \| i k v+\mathrm{e}_{1} \wedge v-\mathrm{e}_{1} \wedge x & \cdot \nabla v\left\|_{q}+\right\| \nabla^{2} v\left\|_{q}+\lambda\right\| \partial_{1} v \|_{q} \\
& +\lambda^{1 / 2}\|v\|_{s_{1}}+\lambda^{1 / 4}\|\nabla v\|_{s_{2}}+\|\nabla p\|_{q} \leq C_{8}\|F\|_{q} .
\end{aligned}
$$

Additionally, if $(w, \mathfrak{q}) \in \mathscr{S}\left(\mathbb{R}^{3}\right)^{3+1}$ is another solution to $(5.1)$ with $w \in \mathrm{L}^{r}(\Omega)$ for some $r \in[1, \infty)$, then $v=w$, and $p-\mathfrak{q}$ is constant.

Proof. First consider a solution $(v, p)$ in the described function class. Then the fields

$$
u(t, x):=\mathrm{e}^{i k t} v(x), \quad \mathfrak{p}(t, x):=\mathrm{e}^{i k t} p(x), \quad f(t, x):=\mathrm{e}^{i k t} F(x),
$$

satisfy (5.5). Therefore, uniqueness of $(v, \nabla p)$ follows from the uniqueness statement in Theorem 5.3. To show existence, let $F \in \mathrm{L}^{q}\left(\mathbb{R}^{3}\right)$ and define $f \in \mathrm{L}^{q}\left(\mathbb{T} \times \mathbb{R}^{3}\right)$ as above. Theorem 5.3 yields the existence of a pair $(u, \mathfrak{p})$ that solves $(5.5)$. Then the $k$-th Fourier coefficients $v(x):=\mathscr{F}_{\mathbb{T}}[u(\cdot, x)](k)$ and $p(x):=$ $\mathscr{F}_{\mathbb{T}}[\mathfrak{p}(\cdot, x)](k)$ satisfy $(5.7)$, and estimate $(5.8)$ is a direct consequence of (5.6). 


\subsection{Uniqueness}

Next we show a uniqueness result for the resolvent problem (2.3).

Lemma 5.6. Let $\lambda \geq 0, \omega>0, k \in \mathbb{Z}$, and let $(v, p)$ be a distributional solution to $(2.3)$ with $F=0$ and $\nabla^{2} v, \partial_{1} v, \nabla p \in \mathrm{L}^{q}(\Omega)$ for some $q \in(1, \infty)$ and $v \in \mathrm{L}^{s}(\Omega)$ for some $s \in(1, \infty)$. Then $v=0$ and $p$ is constant.

Proof. We only consider the case $\lambda>0$ here. The proof for $\lambda=0$ can be shown in exactly the same way. Fix a radius $R>0$ such that $\partial \mathrm{B}_{R} \subset \Omega$, and define a "cut-off" function $\chi_{0} \in \mathrm{C}_{0}^{\infty}\left(\mathbb{R}^{3}\right)$ with $\chi_{0}(x)=1$ for $|x| \leq 2 R$ and $\chi_{0}(x)=0$ for $|x| \geq 4 R$. Set

$$
w:=\chi_{0} v-\mathfrak{B}\left(v \cdot \nabla \chi_{0}\right), \quad \mathfrak{q}:=\chi_{0} p
$$

where $\mathfrak{B}$ denotes the Bogovskiu operator; see for example [13, Section III.3]. Then

$$
\left\{\begin{aligned}
-\Delta w+\nabla \mathfrak{q}=h & \text { in } \Omega_{4 R}, \\
\operatorname{div} w=0 & \text { in } \Omega_{4 R}, \\
w=0 & \text { on } \partial \Omega_{4 R},
\end{aligned}\right.
$$

with

$$
\begin{aligned}
h:= & \left(-\omega\left(i k v+\mathrm{e}_{1} \wedge v-\mathrm{e}_{1} \wedge x \cdot \nabla v\right)-\lambda \partial_{1} v\right) \chi_{0} \\
& -2 \nabla \chi_{0} \cdot \nabla v-\Delta \chi_{0} v+\nabla \chi_{0} p+\Delta \mathfrak{B}\left(\nabla \chi_{0} \cdot v\right) .
\end{aligned}
$$

From the assumptions, we obtain $v \in \mathrm{W}^{2, q}\left(\Omega_{4 R}\right)$ and $p \in \mathrm{W}^{1, q}\left(\Omega_{4 R}\right)$. Standard Sobolev embeddings imply $v, \nabla v, p \in \mathrm{L}^{\frac{3}{2} q}\left(\Omega_{4 R}\right)$. Therefore, we also have $h \in \mathrm{L}^{r}\left(\Omega_{4 R}\right)$ for all $1<r \leq \frac{3}{2} q$. From well-known regularity results for the Stokes problem in bounded domains (see [13, Theorem IV.6.1]) we obtain $w \in \mathrm{W}^{2, r}\left(\Omega_{4 R}\right)$ and $\nabla \mathfrak{q} \in \mathrm{L}^{r}\left(\Omega_{4 R}\right)$. Since $v=w$ and $p=\mathfrak{q}$ on $\Omega_{2 R}$, this yields

$$
(v, p) \in \mathrm{W}^{2, r}\left(\Omega_{2 R}\right) \times \mathrm{W}^{1, r}\left(\Omega_{2 R}\right)
$$

for all $1<r \leq \frac{3}{2} q$.

Next consider another "cut-off" function $\chi_{1} \in \mathrm{C}^{\infty}\left(\mathbb{R}^{3}\right)$ with $\chi_{1}(x)=1$ for $|x| \geq 2 R$ and $\chi_{1}(x)=0$ for $|x| \leq R$. As above, we define

$$
u:=\chi_{1} v-\mathfrak{B}\left(v \cdot \nabla \chi_{1}\right), \quad \mathfrak{p}:=\chi_{1} p
$$

which satisfy the system

$$
\left\{\begin{aligned}
\omega\left(i k u+\mathrm{e}_{1} \wedge u-\mathrm{e}_{1} \wedge x \cdot \nabla u\right)-\Delta u-\lambda \partial_{1} u+\nabla \mathfrak{p}=f & \text { in } \mathbb{R}^{3}, \\
\operatorname{div} u=0 & \text { in } \mathbb{R}^{3},
\end{aligned}\right.
$$

with

$$
\begin{aligned}
f & :=\omega\left(\mathrm{e}_{1} \wedge x \cdot \nabla \chi_{1}\right) v-2 \nabla \chi_{1} \cdot \nabla v-\Delta \chi_{1} v+\lambda \partial_{1} \chi_{1} v+\nabla \chi_{1} p-\Delta \mathfrak{B}\left(v \cdot \nabla \chi_{1}\right) \\
& +\lambda \partial_{1} \mathfrak{B}\left(v \cdot \nabla \chi_{1}\right)+\omega\left(i k \mathfrak{B}\left(v \cdot \nabla \chi_{1}\right)+\mathrm{e}_{1} \wedge \mathfrak{B}\left(v \cdot \nabla \chi_{1}\right)-\mathrm{e}_{1} \wedge x \cdot \nabla \mathfrak{B}\left(v \cdot \nabla \chi_{1}\right)\right) .
\end{aligned}
$$

As above, we see $f \in \mathrm{L}^{r}\left(\mathbb{R}^{3}\right)$ for all $1<r \leq \frac{3}{2} q$. Since we also have $u \in \mathrm{L}^{s}\left(\mathbb{R}^{3}\right)$, Theorem 5.5 implies

$$
i k u+\mathrm{e}_{1} \wedge u-\mathrm{e}_{1} \wedge x \cdot \nabla u, \nabla^{2} u, \partial_{1} u, \nabla \mathfrak{p} \in \mathrm{L}^{r}\left(\mathbb{R}^{3}\right)
$$

if additionally $r<2$. Due to $v=u$ and $p=\mathfrak{p}$ on $\mathrm{B}^{2 R}$, we have

$$
i k v+\mathrm{e}_{1} \wedge v-\mathrm{e}_{1} \wedge x \cdot \nabla v, \nabla^{2} v, \partial_{1} v, \nabla p \in \mathrm{L}^{r}\left(\mathrm{~B}^{2 R}\right)
$$

for $1<r \leq \frac{3}{2} q$ with $r<2$.

We combine (5.10) and (5.13) to deduce

$$
i k v+\mathrm{e}_{1} \wedge v-\mathrm{e}_{1} \wedge x \cdot \nabla v, \nabla^{2} v, \partial_{1} v, \nabla p \in \mathrm{L}^{r}(\Omega)
$$


for $1<r \leq \frac{3}{2} q$ with $r<2$. After repeating the above argument a sufficient number of times, we obtain (5.14) for all $r \in(1,2)$. Since $v \in \mathrm{L}^{s}(\Omega)$, the Sobolev inequality further yields

$$
\forall r \in\left(\frac{3}{2}, 6\right): \nabla v \in \mathrm{L}^{r}(\Omega), \quad \forall r \in(3, \infty): v \in \mathrm{L}^{r}(\Omega) .
$$

In particular, we can employ the divergence theorem to compute

$$
\int_{\Omega_{R}} \operatorname{div}\left[\left(\mathrm{e}_{1} \wedge x\right)|v|^{2}\right] \mathrm{d} x=\int_{\partial \Omega_{R}}\left(\mathrm{e}_{1} \wedge x\right) \cdot \mathrm{n}|v|^{2} \mathrm{~d} S=\int_{\partial \mathrm{B}_{R}}\left(\mathrm{e}_{1} \wedge x\right) \cdot x R^{-1}|v|^{2} \mathrm{~d} S=0
$$

for any $R>0$ with $\partial \mathrm{B}_{R} \subset \Omega$. Passing to the limit $R \rightarrow \infty$, we obtain

$$
\int_{\Omega} \operatorname{div}\left[\left(\mathrm{e}_{1} \wedge x\right)|v|^{2}\right] \mathrm{d} x=0 .
$$

By the above integrability properties, we can further multiply $(2.3)_{1}$ by $v$ and integrate over $\Omega$. By utilizing (5.15) and integration by parts, we conclude

$$
\begin{aligned}
0 & =\int_{\Omega}\left(\omega\left(i k v+\mathrm{e}_{1} \wedge v-\mathrm{e}_{1} \wedge x \cdot \nabla v\right)-\Delta v+\lambda \partial_{1} v+\nabla p\right) \cdot v \mathrm{~d} x \\
& =\int_{\Omega} \omega i k|v|^{2}+\frac{1}{2} \omega \operatorname{div}\left[\left(\mathrm{e}_{1} \wedge x\right)|v|^{2}\right]-\Delta v \cdot v+\frac{1}{2} \lambda \partial_{1}|v|^{2}+\nabla p \cdot v \mathrm{~d} x \\
& =\omega i k \int_{\Omega}|v|^{2} \mathrm{~d} x+\int_{\Omega}|\nabla v|^{2} \mathrm{~d} x .
\end{aligned}
$$

This implies $\nabla v=0$. The imposed boundary conditions thus yield $v=0$. Finally, $(2.3)_{1}$ leads to $\nabla p=0$, and the proof is complete.

\subsection{A Priori Estimate}

Next we establish an a priori estimate for the solution to the resolvent problem (2.3).

Lemma 5.7. Let $q \in(1,2), k \in \mathbb{Z}$ and $\lambda, \omega, \theta>0$ with $\lambda^{2} \leq \theta \omega$. Moreover, let $F \in \mathrm{L}^{q}(\Omega)$ and $R>0$ such that $\partial \mathrm{B}_{R} \subset \Omega$. Let $(v, p) \in \mathrm{L}_{\mathrm{loc}}^{1}(\Omega)$ with

$$
\begin{gathered}
i k v+\mathrm{e}_{1} \wedge v-\mathrm{e}_{1} \wedge x \cdot \nabla v, \nabla^{2} v, \partial_{1} v, \nabla p \in \mathrm{L}^{q}(\Omega), \\
v \in \mathrm{L}^{s_{1}}(\Omega), \quad \nabla v \in \mathrm{L}^{s_{2}}(\Omega)
\end{gathered}
$$

be a solution to (2.3). Then there exists a constant $C_{9}=C_{9}(\Omega, q, \theta, R)>0$ such that

$$
\begin{aligned}
& \omega\left\|i k v+\mathrm{e}_{1} \wedge v-\mathrm{e}_{1} \wedge x \cdot \nabla v\right\|_{q}+\left\|\nabla^{2} v\right\|_{q} \\
& +\lambda\left\|\partial_{1} v\right\|_{q}+\lambda^{1 / 2}\|v\|_{s_{1}}+\lambda^{1 / 4}\|\nabla v\|_{s_{2}}+\|\nabla p\|_{q} \\
& \quad \leq C_{9}\left(\|F\|_{q}+(1+\lambda+\omega)\|v\|_{1, q ; \Omega_{4 R}}+\omega|k|\|v\|_{-1, q ; \Omega_{4 R}}+\|p\|_{q ; \Omega_{4 R}}\right) .
\end{aligned}
$$

Proof. Let $\chi_{0}, \chi_{1}$ be the "cut-off" functions from the proof of Lemma 5.6. Define $w \in \mathrm{W}^{2, q}(\Omega)$ and $\mathfrak{q} \in \mathrm{W}^{1, q}(\Omega)$ as in (5.9). Then

$$
\left\{\begin{aligned}
i k w w-\Delta w+\nabla \mathfrak{q}=h & \text { in } \Omega_{4 R} \\
\operatorname{div} w=0 & \text { in } \Omega_{4 R} \\
w=0 & \text { on } \partial \Omega_{4 R}
\end{aligned}\right.
$$

with

$$
\begin{aligned}
h:= & \left(F-\omega\left(\mathrm{e}_{1} \wedge v-\mathrm{e}_{1} \wedge x \cdot \nabla v\right)-\lambda \partial_{1} v\right) \chi_{0} \\
& -2 \nabla \chi_{0} \cdot \nabla v-\Delta \chi_{0} v+\nabla \chi_{0} p-(i k \omega-\Delta) \mathfrak{B}\left(v \cdot \nabla \chi_{0}\right) .
\end{aligned}
$$


Well-known theory for the Stokes resolvent problem (see for example [9]) yields

$$
\begin{aligned}
& \|v\|_{2, q ; \Omega_{2 R}}+\|\nabla p\|_{q ; \Omega_{2 R}} \leq\|w\|_{2, q ; \Omega_{4 R}}+\|\nabla \mathfrak{q}\|_{q ; \Omega_{4 R}} \leq c_{0}\|h\|_{q ; \Omega_{4 R}} \\
& \quad \leq c_{1}\left(\|F\|_{q}+(1+\lambda+\omega)\|v\|_{1, q ; \Omega_{4 R}}+\|p\|_{q ; \Omega_{4 R}}+\omega|k|\left|v \cdot \nabla \chi_{0}\right|_{-1, q ; \Omega_{4 R}}^{*}\right) .
\end{aligned}
$$

In the last estimate we used mapping properties of the Bogovskiu operator (see [13, Section III.3]), namely

$$
\|\nabla \mathfrak{B} h\|_{m, q ; \Omega_{4 R}} \leq c_{2}\|h\|_{m, q ; \Omega_{4 R}}, \quad\|\mathfrak{B} h\|_{q ; \Omega_{4 R}} \leq c_{3}|h|_{-1, q ; \Omega_{4 R}}^{*}
$$

for $m \in \mathbb{N}_{0}$, where

$$
|h|_{-1, q ; D}^{*}:=\sup \left\{\left|\int_{D} h \psi \mathrm{d} x\right| \mid \psi \in \mathrm{C}_{0}^{\infty}\left(\mathbb{R}^{3}\right),\|\nabla \psi\|_{q /(q-1) ; D}=1\right\} .
$$

To estimate the last term in (5.18), we introduce the notation

$$
\bar{\psi}:=\psi-\frac{1}{\left|\Omega_{4 R}\right|} \int_{\Omega_{4 R}} \psi \mathrm{d} x
$$

for $\psi \in \mathrm{C}_{0}^{\infty}\left(\mathbb{R}^{3}\right)$, and we employ that $\operatorname{div} v=0$ in $\Omega$ and $v=0$ on $\partial \Omega$ to deduce the identity

$$
\begin{aligned}
\int_{\Omega_{4 R}} v \cdot \nabla \chi_{0} \psi \mathrm{d} x & =\int_{\Omega_{4 R}} \operatorname{div}\left(v \chi_{0}\right) \psi \mathrm{d} x=-\int_{\Omega_{4 R}} \chi_{0} v \cdot \nabla \bar{\psi} \mathrm{d} x \\
& =\int_{\Omega_{4 R}} \operatorname{div}\left(v \chi_{0}\right) \bar{\psi} \mathrm{d} x=\int_{\Omega_{4 R}} v \cdot \nabla \chi_{0} \bar{\psi} \mathrm{d} x .
\end{aligned}
$$

Since Poincaré's inequality yields

$$
\left\|\bar{\psi} \nabla \chi_{0}\right\|_{1, q^{\prime} ; \Omega_{4 R}} \leq c_{4}\|\bar{\psi}\|_{1, q^{\prime} ; \Omega_{4 R}} \leq c_{5}\|\nabla \psi\|_{q^{\prime} ; \Omega_{4 R}},
$$

we have

$$
\begin{aligned}
\mid v & \left.\cdot \nabla \chi_{0}\right|_{-1, q ; \Omega_{4 R}} ^{*} \\
& \leq \sup \left\{\|v\|_{-1, q ; \Omega_{4 R}}\left\|\bar{\psi} \nabla \chi_{0}\right\|_{1, q^{\prime} ; \Omega_{4 R}} \mid \psi \in \mathrm{C}_{0}^{\infty}\left(\mathbb{R}^{3}\right),\|\nabla \psi\|_{q^{\prime} ; \Omega_{4 R}}=1\right\} \\
& \leq c_{6}\|v\|_{-1, q ; \Omega_{4 R}} .
\end{aligned}
$$

Applying this estimate to the last term in (5.18), we obtain

$$
\begin{aligned}
& \|v\|_{2, q ; \Omega_{2 R}}+\|\nabla p\|_{q ; \Omega_{2 R}} \\
& \quad \leq c_{7}\left(\|F\|_{q}+(1+\lambda+\omega)\|v\|_{1, q ; \Omega_{4 R}}+\|p\|_{q ; \Omega_{4 R}}+\omega|k|\|v\|_{-1, q ; \Omega_{4 R}}\right) .
\end{aligned}
$$

Next define $(u, \mathfrak{p})$ as in (5.11), which satisfies the system

$$
\left\{\begin{aligned}
\omega\left(i k u+\mathrm{e}_{1} \wedge u-\mathrm{e}_{1} \wedge x \cdot \nabla u\right)-\Delta u-\lambda \partial_{1} u+\nabla \mathfrak{p}=f & \text { in } \mathbb{R}^{3}, \\
\operatorname{div} u=0 & \text { in } \mathbb{R}^{3},
\end{aligned}\right.
$$

with

$$
\begin{aligned}
f:= & \chi_{1} F-\omega\left(\mathrm{e}_{1} \wedge x \cdot \nabla \chi_{1}\right) v-2 \nabla \chi_{1} \cdot \nabla u-\Delta \chi_{1} v \\
& +\lambda \partial_{1} \chi_{1} v+\nabla \chi_{1} p-\Delta \mathfrak{B}\left(v \cdot \nabla \chi_{1}\right)+\lambda \partial_{1} \mathfrak{B}\left(v \cdot \nabla \chi_{1}\right) \\
& +\omega\left(i k \mathfrak{B}\left(v \cdot \nabla \chi_{1}\right)+\mathrm{e}_{1} \wedge \mathfrak{B}\left(v \cdot \nabla \chi_{1}\right)-\mathrm{e}_{1} \wedge x \cdot \nabla \mathfrak{B}\left(v \cdot \nabla \chi_{1}\right)\right) .
\end{aligned}
$$

Theorem 5.5 implies

$$
\begin{aligned}
& \omega\left\|i k v+\mathrm{e}_{1} \wedge v-\mathrm{e}_{1} \wedge x \cdot \nabla v\right\|_{q ; \Omega^{2 R}}+\left\|\nabla^{2} v\right\|_{q ; \Omega^{2 R}}+\lambda\left\|\partial_{1} v\right\|_{q ; \Omega^{2 R}} \\
& \quad+\lambda^{1 / 4}\|\nabla v\|_{s_{2} ; \Omega^{2 R}}+\lambda^{1 / 2}\|v\|_{s_{1} ; \Omega^{2 R}}+\|\nabla p\|_{q ; \Omega^{2 R}} \\
& \leq \omega\left\|i k u+\mathrm{e}_{1} \wedge u-\mathrm{e}_{1} \wedge x \cdot \nabla u\right\|_{q}+\left\|\nabla^{2} u\right\|_{q}+\lambda\left\|\partial_{1} u\right\|_{q} \\
& \quad+\lambda^{1 / 4}\|\nabla u\|_{s_{2}}+\lambda^{1 / 2}\|u\|_{s_{1}}+\|\nabla \mathfrak{p}\|_{q} \\
& \leq c_{8}\left(\|F\|_{q}+(1+\lambda+\omega)\|v\|_{1, q ; \Omega_{2 R}}+\|p\|_{q ; \Omega_{2 R}}+\omega|k|\|v\|_{-1, q ; \Omega_{2 R}}\right),
\end{aligned}
$$


where we estimated the terms containing the Bogovskil operator as above. Combining this estimate with (5.19), we conclude (5.17).

In the next step we improve estimate (5.17) by showing that the lower-order terms on the righthand side can be omitted. This leads to the desired estimate (2.4) with the asserted dependencies of the constant $C_{1}$.

Lemma 5.8. Let $q \in(1,2), k \in \mathbb{Z}$ and $\lambda, \omega>0$, and let $F \in \mathrm{L}^{q}(\Omega)$. Let $(v, p) \in \mathrm{L}_{\text {loc }}^{1}(\Omega)$ be a solution to (2.3) in the class (5.16). Then estimate (2.4) holds for a constant $C_{1}=C_{1}(\Omega, q, \lambda, \omega)>0$. If $q \in\left(1, \frac{3}{2}\right)$ and $\lambda^{2} \leq \theta \omega \leq B$, then this constant can be chosen independently of $\lambda$ and $\omega$ such that $C_{1}=C_{1}(\Omega, q, \theta, B)$.

Proof. We employ a contradiction argument. At first, consider the case $q \in\left(1, \frac{3}{2}\right)$ and assume that $(2.4)$ is not valid for a constant $C_{1}=C_{1}(\Omega, q, \theta, B)$. Then there exist sequences of numbers $\left(\lambda_{j}\right) \subset(0, \sqrt{B}]$, $\left(\omega_{j}\right) \subset(0, B / \theta]$ with $\lambda_{j}^{2} \leq \theta \omega_{j}$, and $\left(k_{j}\right) \subset \mathbb{Z}$, and of functions $\left(v_{j}\right),\left(p_{j}\right),\left(F_{j}\right)$ that satisfy

$$
\begin{aligned}
& \omega_{j}\left\|i k_{j} v_{j}+\mathrm{e}_{1} \wedge v_{j}-\mathrm{e}_{1} \wedge x \cdot \nabla v_{j}\right\|_{q}+\left\|\nabla^{2} v_{j}\right\|_{q} \\
& +\lambda_{j}\left\|\partial_{1} v_{j}\right\|_{q}+\lambda_{j}^{1 / 2}\left\|v_{j}\right\|_{s_{1}}+\lambda_{j}^{1 / 4}\left\|\nabla v_{j}\right\|_{s_{2}}+\left\|\nabla p_{j}\right\|_{q}=1
\end{aligned}
$$

$\left\|F_{j}\right\|_{q} \rightarrow 0$ as $j \rightarrow \infty$, and

$$
\left\{\begin{aligned}
\omega_{j}\left(i k_{j} v_{j}+\mathrm{e}_{1} \wedge v_{j}-\mathrm{e}_{1} \wedge x \cdot \nabla v_{j}\right)-\Delta v_{j}-\lambda_{j} \partial_{1} v_{j}+\nabla p_{j}=F_{j} & \text { in } \Omega, \\
\operatorname{div} v_{j}=0 & \text { in } \Omega, \\
v_{j}=0 & \text { on } \partial \Omega
\end{aligned}\right.
$$

for all $j \in \mathbb{N}$. Furthermore, without loss of generality, we assume $\int_{\Omega_{R}} p_{j} \mathrm{~d} x=0$ for $R>0$ as in Lemma 5.7. Then, $\left(\lambda_{j}\right),\left(\omega_{j}\right)$ and $\left(k_{j}\right)$ contain (improper) convergent subsequences with limits $\lambda \in[0, \sqrt{B}]$, $\omega \in[0, B / \theta]$ and $k \in \mathbb{Z} \cup\{ \pm \infty\}$, respectively, and we have $\lambda^{2} \leq \theta \omega$. For simplicity, we identify selected subsequences with the actual sequences. Moreover, (5.20) implies that $U_{j}:=\left(i \omega_{j} k_{j} v_{j}, v_{j}, p_{j}\right)$ is bounded in $\mathrm{L}^{q}\left(\Omega_{\rho}\right) \times \mathrm{W}^{2, q}\left(\Omega_{\rho}\right) \times \mathrm{W}^{1, q}\left(\Omega_{\rho}\right)$ for any $\rho>R$. Hence, by a Cantor diagonalization argument, there exists a subsequence that converges weakly in $\mathrm{L}^{q}\left(\Omega_{\rho}\right) \times \mathrm{W}^{2, q}\left(\Omega_{\rho}\right) \times \mathrm{W}^{1, q}\left(\Omega_{\rho}\right)$ to some $U:=(w, v, p)$ for each $\rho>R$. Consequently, passing to the limit $j \rightarrow \infty$ in (5.21), we obtain

$$
\left\{\begin{aligned}
w+\omega\left(\mathrm{e}_{1} \wedge v-\mathrm{e}_{1} \wedge x \cdot \nabla v\right)-\Delta v-\lambda \partial_{1} v+\nabla \mathfrak{p}=0 & & \text { in } \Omega \\
\operatorname{div} v=0 & & \text { in } \Omega \\
v=0 & & \text { on } \partial \Omega .
\end{aligned}\right.
$$

Moreover, by the compact embeddings

$$
\mathrm{W}^{2, q}\left(\Omega_{4 R}\right) \hookrightarrow \mathrm{W}^{1, q}\left(\Omega_{4 R}\right) \hookrightarrow \mathrm{L}^{q}\left(\Omega_{4 R}\right) \hookrightarrow \mathrm{W}^{-1, q}\left(\Omega_{4 R}\right)
$$

we deduce that $U$ is the strong limit of $\left(U_{j}\right)$ in the topology of $\mathrm{W}^{-1, q}\left(\Omega_{4 R}\right) \times \mathrm{W}^{1, q}\left(\Omega_{4 R}\right) \times \mathrm{L}^{q}\left(\Omega_{4 R}\right)$. By Lemma 5.7,

$$
\begin{aligned}
& \omega_{j}\left\|i k_{j} v_{j}+\mathrm{e}_{1} \wedge v_{j}-\mathrm{e}_{1} \wedge x \cdot \nabla v_{j}\right\|_{q}+\left\|\nabla^{2} v_{j}\right\|_{q} \\
& \quad+\lambda_{j}\left\|\partial_{1} v_{j}\right\|_{q}+\lambda_{j}^{1 / 2}\left\|v_{j}\right\|_{s_{1}}+\lambda_{j}^{1 / 4}\left\|\nabla v_{j}\right\|_{s_{2}}+\left\|\nabla p_{j}\right\|_{q} \\
& \quad \leq C_{9}\left(\left\|F_{j}\right\|_{q}+\left(1+\lambda_{j}+\omega_{j}\right)\left\|v_{j}\right\|_{1, q ; \Omega_{4 R}}+\omega\left|k_{j}\right|\left\|v_{j}\right\|_{-1, q ; \Omega_{4 R}}+\left\|p_{j}\right\|_{q ; \Omega_{4 R}}\right) .
\end{aligned}
$$

Passing to the limit $j \rightarrow \infty$ in this estimate, we conclude in virtue of (5.20) that

$$
1 \leq C_{9}\left((1+\lambda+\omega)\|v\|_{1, q ; \Omega_{4 R}}+\|w\|_{-1, q ; \Omega_{4 R}}+\|p\|_{q ; \Omega_{4 R}}\right) .
$$

Moreover,

$$
\begin{array}{r}
\left\|w+\omega\left(\mathrm{e}_{1} \wedge v-\mathrm{e}_{1} \wedge x \cdot \nabla v\right)\right\|_{q}+\left\|\nabla^{2} v\right\|_{q}+\lambda\left\|\partial_{1} v\right\|_{q} \\
+\lambda^{1 / 2}\|v\|_{s_{1}}+\lambda^{1 / 4}\|\nabla v\|_{s_{2}}+\|\nabla p\|_{q}<\infty .
\end{array}
$$

Now we distinguish between several cases: 
i. If $\omega_{j} k_{j} \rightarrow s \in \mathbb{R}$ and $\omega=0$, then $\lambda=0$ and $w=i s v$, so that (5.22) reduces to a Stokes resolvent problem. If $s \neq 0$, we also have $v \in \mathrm{L}^{q}(\Omega)$ and we conclude $v=\nabla p=0$ from a well-known uniqueness result; see for example [9]. If $s=0$, we utilize that $q<\frac{3}{2}$ and $v_{j} \in \mathrm{L}^{s_{1}}(\Omega), \nabla v_{j} \in \mathrm{L}^{s_{2}}(\Omega)$, so that Sobolev's inequality implies

$$
\left\|v_{j}\right\|_{3 q /(3-2 q)} \leq c_{0}\left\|\nabla v_{j}\right\|_{3 q /(3-q)} \leq c_{1}\left\|\nabla^{2} v_{j}\right\|_{q},
$$

and thus $v \in \mathrm{L}^{3 q /(3-2 q)}(\Omega)$. Now $v=\nabla p=0$ follows from classical uniqueness properties of the steady-state Stokes problem, see for example [13, Theorem V.4.6].

ii. If $\omega_{j} k_{j} \rightarrow s \in \mathbb{R}$ and $\omega \neq 0$ but $\lambda=0$, then $k_{j} \rightarrow k \in \mathbb{Z}$ and $w=i \omega k v$, so that (5.22) reduces to (2.3) with $\lambda=0$. As above, we deduce $v \in \mathrm{L}^{3 q /(3-2 q)}(\Omega)$. From Lemma 5.6 we conclude $v=\nabla p=0$. iii. If $\omega_{j} k_{j} \rightarrow s \in \mathbb{R}$ and $\omega \neq 0$ and $\lambda \neq 0$, then $k_{j} \rightarrow k \in \mathbb{Z}$ and $w=i \omega k v$, so that $(v, p)$ satisfies $(2.3)$. Since $\lambda \neq 0$, it follows from (5.24) that $v \in \mathrm{L}^{s_{1}}(\Omega)$. Lemma 5.6 thus implies $v=\nabla p=0$.

iv. If $\omega_{j}\left|k_{j}\right| \rightarrow \infty$, we recall (5.20) and estimate

$$
\omega_{j}\left|k_{j}\right|\left\|v_{j}\right\|_{q ; \Omega_{\rho}} \leq \omega_{j}\left\|i k_{j} v_{j}+\mathrm{e}_{1} \wedge v_{j}-\mathrm{e}_{1} \wedge x \cdot \nabla v_{j}\right\|_{q ; \Omega_{\rho}}+c_{2}(\rho)\left\|v_{j}\right\|_{1, q ; \Omega_{\rho}} \leq c_{3}(\rho)
$$

for any $\rho>R$. Passing to the limit $j \rightarrow \infty$, we thus obtain $v=0$ on $\Omega_{\rho}$ for each $\rho>R$, whence $v=0$ on $\Omega$. Hence, $(5.22)_{1}$ reduces to $w+\nabla p=0$. Clearly, we also have $\operatorname{div} w=0$ and $\left.w\right|_{\partial \Omega}=0$, so that $w+\nabla p=0$ corresponds to the Helmholtz decomposition of 0 in $\mathrm{L}^{q}(\Omega)$. Since this decomposition is unique, we conclude $w=\nabla p=0$.

Consequently, all four cases lead to $w=v=\nabla p=0$, which contradicts (5.23). This completes the proof in the case $1<q<\frac{3}{2}$.

In the more general case $q \in(1,2)$, where we do not assert the constant $C_{1}$ to be independent of $\lambda$ and $\omega$, these parameters remain fixed in the contradiction argument above. Consequently, only the last two cases above have to be considered. The conclusion in both of these cases is valid for all $q \in(1,2)$, and we thus conclude the lemma.

\subsection{Existence}

To complete the proof of Theorem 2.1, it remains to show existence of a solution. For this purpose, recall the following property of the Stokes operator.

Lemma 5.9. Let $D \subset \mathbb{R}^{3}$ be a bounded domain with $\mathrm{C}^{3}$-boundary. Every $u \in \mathrm{L}_{\sigma}^{2}(D) \cap \mathrm{W}_{0}^{1,2}(D) \cap \mathrm{W}^{2,2}(D)$ satisfies

$$
\left\|\nabla^{2} u\right\|_{2} \leq C_{10}\left(\left\|\mathcal{P}_{\mathrm{H}} \Delta u\right\|_{2}+\|\nabla u\|_{2}\right)
$$

for a constant $C_{10}=C_{10}(D)>0$ that does not depend on the "size" of D but solely on its "regularity". In particular, if $D=\Omega_{R}$ for an exterior domain $\Omega$ with $\partial \Omega \subset \mathrm{B}_{R}$, the constant $C_{10}$ is independent of $R$ and solely depends on $\Omega$.

Proof. See [26, Lemma 1].

We further need the following identity from [20].

Lemma 5.10. Let $u \in \mathrm{L}_{\sigma}^{2}\left(\Omega_{R}\right) \cap \mathrm{W}_{0}^{1,2}\left(\Omega_{R}\right) \cap \mathrm{W}^{2,2}\left(\Omega_{R}\right)$ with complex conjugate $u^{*}$. Then $\mathrm{e}_{1} \wedge u-\mathrm{e}_{1} \wedge x \cdot \nabla u \in$ $\mathrm{L}_{\sigma}^{2}\left(\Omega_{R}\right)$ and

$$
\begin{aligned}
& \int_{\Omega_{R}}\left(\mathrm{e}_{1} \wedge u-\mathrm{e}_{1} \wedge x \cdot \nabla u\right) \cdot \mathcal{P}_{\mathrm{H}} \Delta u^{*} \mathrm{~d} x \\
& =\int_{\partial \Omega} \frac{1}{2}|\nabla u|^{2}\left(\mathrm{e}_{1} \wedge x\right) \cdot \mathrm{n}-\mathrm{n} \cdot \nabla u^{*} \cdot\left(\mathrm{e}_{1} \wedge x \cdot \nabla u\right) \mathrm{d} S-\int_{\Omega_{R}} \nabla\left(\mathrm{e}_{1} \wedge u\right): \nabla u^{*} \mathrm{~d} x .
\end{aligned}
$$

Proof. See [20, Lemma 3]. 
Existence of a solution to the resolvent problem (2.3) can be shown via a Galerkin approach combined with an "invading domains" technique.

Lemma 5.11. Let $\Omega \subset \mathbb{R}^{3}$ be an exterior domain of class $\mathrm{C}^{3}$. Let $\lambda, \omega>0, k \in \mathbb{Z}$, and let $F \in \mathrm{C}_{0}^{\infty}(\Omega)$. Then there exists a solution $(v, p)$ to $(2.3)$ with

$$
\begin{aligned}
& i k v+\mathrm{e}_{1} \wedge v-\mathrm{e}_{1} \wedge x \cdot \nabla v, \nabla^{2} v, \partial_{1} v, \nabla p \in \mathrm{L}^{q}(\Omega), \\
& v \in \mathrm{L}^{2 q /(2-q)}(\Omega), \quad \nabla v \in \mathrm{L}^{4 q /(4-q)}(\Omega)
\end{aligned}
$$

for all $q \in(1,2)$.

Proof. Let $R>0$ such that $\partial \mathrm{B}_{R} \subset \Omega$, and take $m \in \mathbb{N}$ with $m>2 R$. Since the Stokes operator in the bounded domain $\Omega_{m}$ is a positive self-adjoint invertible operator (see [50, Chapter III, Theorem 2.1.1]), there exist sequences $\left(\psi_{j}\right)_{j \in \mathbb{N}}$ of (real-valued) eigenfunctions and $\left(\mu_{j}\right)_{j \in \mathbb{N}} \subset(0, \infty)$ of eigenvalues, that is,

$$
-\mathcal{P}_{\mathrm{H}} \Delta \psi_{j}=\mu_{j} \psi_{j}, \quad \psi_{j} \in \mathrm{L}_{\sigma}^{2}\left(\Omega_{m}\right) \cap \mathrm{W}_{0}^{1,2}\left(\Omega_{m}\right) \cap \mathrm{W}^{2,2}\left(\Omega_{m}\right),
$$

normalized such that

$$
\int_{\Omega_{m}} \psi_{j} \cdot \psi_{\ell} \mathrm{d} x=\frac{1}{\mu_{j}} \delta_{j \ell}
$$

We show the existence of a function $u=u_{n}^{m} \in X_{n}^{m}:=\operatorname{span}_{\mathbb{C}}\left\{\psi_{j} \mid j=1, \ldots, n\right\}$ satisfying

$$
\int_{\Omega_{m}}\left[\omega\left(i k u+\mathrm{e}_{1} \wedge u-\mathrm{e}_{1} \wedge x \cdot \nabla u\right)-\Delta u-\lambda \partial_{1} u\right] \cdot \psi_{j} \mathrm{~d} x=\int_{\Omega_{m}} F \cdot \psi_{j} \mathrm{~d} x
$$

for all $j \in\{1, \ldots, n\}$. Since

$$
u=\sum_{\ell=1}^{n} \xi_{\ell} \psi_{\ell}
$$

for some $\xi_{1}, \ldots, \xi_{n} \in \mathbb{C}$, this is equivalent to solving the algebraic equation

$$
(\mathrm{I}+M) \xi=c
$$

with $\xi=\left(\xi_{1}, \ldots, \xi_{n}\right) \in \mathbb{C}^{n}$ and $M=\left(M_{\ell j}\right) \in \mathbb{C}^{n \times n}, c=\left(c_{j}\right) \in \mathbb{C}^{n}$ with

$$
\begin{aligned}
M_{\ell j} & :=\int_{\Omega_{m}}\left(\omega\left(i k \psi_{\ell}+\mathrm{e}_{1} \wedge \psi_{\ell}-\mathrm{e}_{1} \wedge x \cdot \nabla \psi_{\ell}\right)-\lambda \partial_{1} \psi_{\ell}\right) \cdot \psi_{j} \mathrm{~d} x, \\
c_{j} & :=\int_{\Omega_{m}} F \cdot \psi_{j} \mathrm{~d} x .
\end{aligned}
$$

Note that (5.26) is a resolvent problem for the skew-Hermitian matrix $M$, which is uniquely solvable. Existence of a unique solution $u=u_{n}^{m} \in X_{n}^{m}$ to (5.25) thus follows.

Next we need suitable estimates for $u=u_{n}^{m}$. Multiplication of both sides of (5.25) by the complex conjugate coefficient $\xi_{j}^{*}$ and summation over $j=1, \ldots, n$ yields

$$
\|\nabla u\|_{2}^{2}+\int_{\Omega_{m}}\left(\omega\left(i k u+\mathrm{e}_{1} \wedge u-\mathrm{e}_{1} \wedge x \cdot \nabla u\right)-\lambda \partial_{1} u\right) \cdot u^{*} \mathrm{~d} x=\int_{\Omega_{m}} F \cdot u^{*} \mathrm{~d} x .
$$

Because the integral term on the left-hand side is purely imaginary, taking the real part of this equation leads to the estimate

$$
\|\nabla u\|_{2}^{2} \leq\|F\|_{6 / 5}\|u\|_{6} .
$$

Recalling the Sobolev inequality $\|u\|_{6} \leq c_{0}\|\nabla u\|_{2}$, we obtain

$$
\|u\|_{6}+\|\nabla u\|_{2} \leq c_{1}\|F\|_{6 / 5}
$$


where $c_{1}$ is independent of $m$. If we multiply both sides of $(5.25)$ by $\mu_{j} \xi_{j}^{*}$ and sum over $j=1, \ldots, n$, we obtain

$$
\left\|\mathcal{P}_{\mathrm{H}} \Delta u\right\|_{2}^{2}=\int_{\Omega_{m}}\left[F-\omega\left(i k u+\mathrm{e}_{1} \wedge u-\mathrm{e}_{1} \wedge x \cdot \nabla u\right)+\lambda \partial_{1} u\right] \cdot \mathcal{P}_{\mathrm{H}} \Delta u^{*} \mathrm{~d} x .
$$

Taking real part of both sides and observing that

$$
\operatorname{Re} \int_{\Omega_{m}} i k u \cdot \mathcal{P}_{\mathrm{H}} \Delta u^{*} \mathrm{~d} x=-\operatorname{Re}\left(i k\|\nabla u\|_{2}^{2}\right)=0,
$$

we conclude, using Hölder's inequality, the estimate

$$
\begin{aligned}
& \left\|\mathcal{P}_{\mathrm{H}} \Delta u\right\|_{2}^{2} \leq\left(\|F\|_{2}+\lambda\left\|\partial_{1} u\right\|_{2}\right)\left\|\mathcal{P}_{\mathrm{H}} \Delta u\right\|_{2} \\
& \quad+\operatorname{Re} \int_{\Omega_{m}} \omega\left(\mathrm{e}_{1} \wedge u-\mathrm{e}_{1} \wedge x \cdot \nabla u\right) \cdot \mathcal{P}_{\mathrm{H}} \Delta u^{*} \mathrm{~d} x .
\end{aligned}
$$

Using Lemma 5.10, we estimate the remaining integral on the right-hand side to conclude

$$
\operatorname{Re} \int_{\Omega_{m}} \omega\left(\mathrm{e}_{1} \wedge u-\mathrm{e}_{1} \wedge x \cdot \nabla u\right) \cdot \mathcal{P}_{\mathrm{H}} \Delta u^{*} \mathrm{~d} x \leq c_{2} \omega\left(\|\nabla u\|_{2 ; \partial \Omega}^{2}+\|\nabla u\|_{2 ; \Omega_{m}}^{2}\right)
$$

with $c_{2}$ independent of $m$. Employing the trace inequality [13, Theorem II.4.1] on the domain $\Omega_{R}$, we further estimate

$$
\begin{aligned}
\operatorname{Re} \int_{\Omega_{m}} & \omega\left(\mathrm{e}_{1} \wedge u-\mathrm{e}_{1} \wedge x \cdot \nabla u\right) \cdot \mathcal{P}_{\mathrm{H}} \Delta u^{*} \mathrm{~d} x \\
& \leq c_{3} \omega\left(\|\nabla u\|_{2 ; \Omega_{R}}\|\nabla u\|_{1,2 ; \Omega_{R}}+\|\nabla u\|_{2 ; \Omega_{m}}^{2}\right) \\
& \leq c_{4}(\varepsilon)\left(\omega+\omega^{2}\right)\|\nabla u\|_{2 ; \Omega_{m}}^{2}+\varepsilon\left\|\nabla^{2} u\right\|_{2 ; \Omega_{m}}^{2}
\end{aligned}
$$

for small $\varepsilon>0$. From Lemma 5.9 we deduce

$$
\begin{aligned}
& \operatorname{Re} \int_{\Omega_{m}} \omega\left(\mathrm{e}_{1} \wedge u-\mathrm{e}_{1} \wedge x \cdot \nabla u\right) \cdot \mathcal{P}_{\mathrm{H}} \Delta u^{*} \mathrm{~d} x \\
& \quad \leq c_{5}(\varepsilon)\left(\omega+\omega^{2}\right)\|\nabla u\|_{2 ; \Omega_{m}}^{2}+\varepsilon c_{6}\left\|\mathcal{P}_{\mathrm{H}} \Delta u\right\|_{2 ; \Omega_{m}}^{2}
\end{aligned}
$$

with a constant $c_{6}>0$ independent of $m$. Combining this estimate with (5.28), choosing $\varepsilon$ sufficiently small and employing estimate (5.27), we arrive at

$$
\left\|\mathcal{P}_{\mathrm{H}} \Delta u\right\|_{2 ; \Omega_{m}} \leq c_{7}\left(1+\lambda+\sqrt{\omega+\omega^{2}}\right)\left(\|F\|_{2}+\|F\|_{6 / 5}\right) .
$$

Using Lemma 5.9 and estimate (5.27) once again and restoring the original notation, we end up with

$$
\left\|\nabla^{2} u_{n}^{m}\right\|_{2 ; \Omega_{m}} \leq c_{8}\left(\left\|\mathcal{P}_{\mathrm{H}} \Delta u_{n}^{m}\right\|_{2 ; \Omega_{m}}+\left\|\nabla u_{n}^{m}\right\|_{2 ; \Omega_{m}}\right) \leq c_{9}\left(\|F\|_{2}+\|F\|_{6 / 5}\right)
$$

with $c_{9}$ independent of $m$.

In particular, we see from (5.27), (5.29) and Poincaré's inequality that $\left(u_{n}^{m}\right)$ is uniformly bounded in $\mathrm{W}^{2,2}\left(\Omega_{m}\right)$ and thus contains a subsequence that converges weakly to some function $v^{m} \in \mathrm{L}_{\sigma}^{2}\left(\Omega_{m}\right) \cap$ $\mathrm{W}_{0}^{1,2}\left(\Omega_{m}\right) \cap \mathrm{W}^{2,2}\left(\Omega_{m}\right)$, which obeys the estimate

$$
\left\|v^{m}\right\|_{6 ; \Omega_{m}}+\left\|\nabla v^{m}\right\|_{1,2 ; \Omega_{m}} \leq c_{10}\left(\|F\|_{6 / 5}+\|F\|_{2}\right)
$$

with $c_{10}$ independent of $m$. Moreover, $v^{m}$ satisfies (5.25) for all $j \in \mathbb{N}$, whence there exists $p^{m} \in \mathrm{W}^{1,2}\left(\Omega_{m}\right)$ such that

$$
\left\{\begin{aligned}
\omega\left(i k v^{m}+\mathrm{e}_{1} \wedge v^{m}-\mathrm{e}_{1} \wedge x \cdot \nabla v^{m}\right)-\Delta v^{m}-\lambda \partial_{1} v^{m}+\nabla p^{m}=F & \text { in } \Omega_{m}, \\
\operatorname{div} v^{m}=0 & \text { in } \Omega_{m}, \\
v^{m}=0 & \text { on } \partial \Omega_{m}
\end{aligned}\right.
$$

see [13, Corollary III.5.1]. Since $\mathrm{e}_{1} \wedge v^{m}-\mathrm{e}_{1} \wedge x \cdot \nabla v^{m} \in \mathrm{L}_{\sigma}^{2}\left(\Omega_{m}\right)$ by Lemma 5.10, we deduce from (5.31) and (5.30) the estimate

$$
\omega\left\|i k v^{m}+\mathrm{e}_{1} \wedge v^{m}-\mathrm{e}_{1} \wedge x \cdot \nabla v^{m}\right\|_{2}=\omega\left\|\mathcal{P}_{\mathrm{H}}\left(i k v^{m}+\mathrm{e}_{1} \wedge v^{m}-\mathrm{e}_{1} \wedge x \cdot \nabla v^{m}\right)\right\|_{2}
$$




$$
\leq\left\|\mathcal{P}_{\mathrm{H}} F\right\|_{2}+\left\|\mathcal{P}_{\mathrm{H}} \Delta v^{m}\right\|_{2}+\lambda\left\|\mathcal{P}_{\mathrm{H}} \partial_{1} v^{m}\right\|_{2} \leq c_{11}\left(\|F\|_{6 / 5}+\|F\|_{2}\right) .
$$

Combining the estimate above with (5.30), we conclude

$$
\begin{aligned}
\left\|v^{m}\right\|_{6 ; \Omega_{m}}+\left\|\nabla v^{m}\right\|_{1,2 ; \Omega_{m}}+\omega \| i k v^{m}+\mathrm{e}_{1} \wedge v^{m} & -\mathrm{e}_{1} \wedge x \cdot \nabla v^{m} \|_{2 ; \Omega_{m}} \\
& \leq c_{12}\left(\|F\|_{6 / 5}+\|F\|_{2}\right)
\end{aligned}
$$

with $c_{12}$ independent of $m$.

Now we introduce a sequence of rotationally symmetric "cut-off" functions $\left(\chi_{m}\right) \subset \mathrm{C}_{0}^{\infty}\left(\mathbb{R}^{3}\right)$ satisfying

$$
\begin{array}{lll}
\chi_{m}(x)=1 \text { for }|x| \leq \frac{m}{2}, & & \left|\nabla \chi_{m}\right| \leq \frac{c_{13}}{m}, \\
\chi_{m}(x)=0 \text { for }|x| \geq \frac{3 m}{4}, & \left|\nabla^{2} \chi_{m}\right| \leq \frac{c_{14}}{m^{2}},
\end{array}
$$

and we set $w^{m}:=\chi_{m} v^{m}$. Then $w^{m}$ is an element of $\mathrm{W}^{2,2}(\Omega)$. Moreover, the rotational symmetry of $\chi_{m}$ implies $\mathrm{e}_{1} \wedge x \cdot \nabla \chi_{m}=0$. Therefore, from (5.32) and the properties of $\chi_{m}$, we deduce the estimate

$$
\left\|w^{m}\right\|_{6}+\left\|\nabla w^{m}\right\|_{1,2}+\omega\left\|i k w^{m}+\mathrm{e}_{1} \wedge w^{m}-\mathrm{e}_{1} \wedge x \cdot \nabla w^{m}\right\|_{2} \leq c_{15}\left(\|F\|_{6 / 5}+\|F\|_{2}\right)
$$

with $c_{15}$ independent of $m$. This implies the existence of a subsequence, still denoted by $\left(w^{m}\right)$, that converges in the sense of distributions to some function $v \in \mathrm{W}_{\text {loc }}^{2,2}(\Omega)$ that satisfies

$$
\|v\|_{6}+\|\nabla v\|_{1,2}+\omega\left\|i k v+\mathrm{e}_{1} \wedge v-\mathrm{e}_{1} \wedge x \cdot \nabla v\right\|_{2} \leq c_{12}\left(\|F\|_{6 / 5}+\|F\|_{2}\right) .
$$

Moreover, $\left.v\right|_{\partial \Omega}=0$. Let $\varphi \in \mathrm{C}_{0}^{\infty}(\Omega)$. We choose $m_{0} \in \mathbb{N}$ such that $\operatorname{supp} \varphi$ is contained in $\Omega_{m_{0} / 2}$. For $m \geq m_{0}$ we have $w^{m}=v^{m}$ on $\Omega_{m_{0} / 2}$ and thus

$$
\int_{\Omega} w^{m} \cdot \nabla \varphi \mathrm{d} x=\int_{\Omega} v^{m} \cdot \nabla \varphi \mathrm{d} x=0
$$

by $(5.31)_{2}$. Passing to the limit $m \rightarrow \infty$, we conclude $\operatorname{div} v=0$. Now let $\psi \in \mathrm{C}_{0, \sigma}^{\infty}(\Omega)$ and choose $m_{0}$ such that supp $\psi \subset \Omega_{m_{0} / 2}$. With the same argument as above, for $m \geq m_{0}$ we obtain from (5.31) $)_{1}$ that

$$
\begin{aligned}
& \int_{\Omega}\left(\omega\left(i k w^{m}+\mathrm{e}_{1} \wedge w^{m}-\mathrm{e}_{1} \wedge x \cdot \nabla w^{m}\right)-\Delta w^{m}-\lambda \partial_{1} w^{m}-F\right) \cdot \psi \mathrm{d} x \\
& =\int_{\Omega}\left(\omega\left(i k v^{m}+\mathrm{e}_{1} \wedge v^{m}-\mathrm{e}_{1} \wedge x \cdot \nabla v^{m}\right)-\Delta v^{m}-\lambda \partial_{1} v^{m}+\nabla p^{m}-F\right) \cdot \psi \mathrm{d} x=0 .
\end{aligned}
$$

Therefore, by passing to the limit $m \rightarrow \infty$, we see

$$
\int_{\Omega}\left(\omega\left(i k v+\mathrm{e}_{1} \wedge v-\mathrm{e}_{1} \wedge x \cdot \nabla v\right)-\Delta v-\lambda \partial_{1} v-F\right) \cdot \psi \mathrm{d} x=0
$$

for all $\psi \in \mathrm{C}_{0, \sigma}^{\infty}(\Omega)$. Consequently, by the Helmholtz decomposition, there exists a function $p$ with $\nabla p \in \mathrm{L}^{2}(\Omega)$ such that $(v, p)$ is a solution to $(2.3)$.

It remains to show that $v$ and $p$ belong to the correct function spaces. By Hölder's inequality, we directly find that

$$
v \in \mathrm{W}^{2, q}\left(\Omega_{\rho}\right), \quad p \in \mathrm{W}^{1, q}\left(\Omega_{\rho}\right)
$$

for any $\rho>R$ and all $q \in[1,2]$. Repeating the "cut-off" argument from (5.11), we obtain $(u, \mathfrak{p})$ which satisfy (5.12) for some function $f \in \mathrm{L}^{2}\left(\mathbb{R}^{3}\right)$ with compact support. In particular, this implies $f \in \mathrm{L}^{q}\left(\mathbb{R}^{3}\right)$ for all $q \in(1,2)$. Theorem 5.5 yields existence of a solution to (5.12) satisfying (5.8). Since $u \in \mathrm{L}^{6}\left(\mathbb{R}^{3}\right)$, Theorem 5.5 further ensures that $(u, \mathfrak{p})$ coincides with this solution. We thus have

$$
\begin{aligned}
& i k u+\mathrm{e}_{1} \wedge u-\mathrm{e}_{1} \wedge x \cdot \nabla u, \nabla^{2} u, \partial_{1} u, \nabla \mathfrak{p} \in \mathrm{L}^{q}\left(\mathbb{R}^{3}\right), \\
& u \in \mathrm{L}^{2 q /(2-q)}\left(\mathbb{R}^{3}\right), \quad \nabla u \in \mathrm{L}^{4 q /(4-q)}\left(\mathbb{R}^{3}\right) .
\end{aligned}
$$

Since $v=u$ and $p=\mathfrak{p}$ on $\mathrm{B}^{2 R}$, the integrability properties above in combination with (5.34) show that $v$ and $p$ belong to the correct function spaces. 
Combining Lemmas 5.6, 5.8 and 5.11, we can finally complete the proof of Theorem 2.1.

Proof of Theorem 2.1. The uniqueness statement is a direct consequence of Lemma 5.6. Estimate (2.4) has been proved in Lemma 5.8. It thus remains to show existence of a solution for $F \in \mathrm{L}^{q}(\Omega)$. Consider a sequence $\left(F_{j}\right) \subset \mathrm{C}_{0}^{\infty}(\Omega)$ that converges to $F$ in $\mathrm{L}^{q}(\Omega)$. By Lemma 5.11, for each $j \in \mathbb{N}$ there exists a solution $(v, p)=\left(v_{j}, p_{j}\right)$ to $(2.3)$ with $F=F_{j}$, which obeys estimate (2.4) by Lemma 5.8. Additionally, this implies that $\left(v_{j}, \nabla p_{j}\right)$ is a Cauchy sequence in the function space defined by the norm on the left-hand side of (2.4), and thus possesses a limit $(v, \nabla p)$, which satisfies (2.3) and (2.4).

\subsection{The Time-Periodic Linear Problem}

Proof of Theorem 2.2. An application of the Fourier transform $\mathscr{F}_{\mathbb{T}}$ on $\mathbb{T}$ to $(2.2)$ reduces the uniqueness statement to the corresponding uniqueness result for the resolvent problem established in Theorem 2.1. To show existence, consider $f \in \mathrm{A}\left(\mathbb{T} ; \mathrm{L}^{q}(\Omega)\right)$. Then

$$
f(t, x)=\sum_{k \in \mathbb{Z}} f_{k}(x) \mathrm{e}^{i k t}
$$

with $f_{k} \in \mathrm{L}^{q}(\Omega)$. Let $\left(u_{k}, \mathfrak{p}_{k}\right)=(v, p)$ be a solution to the resolvent problem (2.3) with $F=f_{k}$ that exists due to Theorem 2.1. We define

$$
u(t, x):=\sum_{k \in \mathbb{Z}} u_{k}(x) \mathrm{e}^{i k t}, \quad \mathfrak{p}(t, x):=\sum_{k \in \mathbb{Z}} \mathfrak{p}_{k}(x) \mathrm{e}^{i k t} .
$$

By (2.4), $u$ and $\mathfrak{p}$ are well defined and satisfy (2.2). We directly conclude estimate (2.5) from estimate $(2.4)$.

\section{The Nonlinear Problem}

We return to the nonlinear problem (2.1). At first, we reformulate it as a problem with homogeneous boundary conditions. To this end, fix $R>0$ such that $\partial \mathrm{B}_{R} \subset \Omega$. Let $\varphi \in \mathrm{C}_{0}^{\infty}\left(\mathbb{R}^{3}\right)$ be a smooth function satisfying $\varphi(x)=1$ if $|x|<R$, and $\varphi(x)=0$ if $|x|>2 R$, and define

$$
U: \mathbb{T} \times \mathbb{R}^{3} \rightarrow \mathbb{R}^{3}, \quad U(t, x)=\frac{1}{2} \operatorname{rot}\left[\left(\alpha(t) \mathrm{e}_{1} \wedge x-\omega \mathrm{e}_{1}|x|^{2}\right) \varphi(x)\right] .
$$

Then $U(t, \cdot) \in \mathrm{C}_{0}^{\infty}\left(\mathbb{R}^{3}\right)^{3}$ for all $t \in \mathbb{T}, U \in \mathrm{C}^{1}\left(\mathbb{T} \times \mathbb{R}^{3}\right)$, div $U=0$, and a brief calculation shows $U(t, x)=\alpha(t) \mathrm{e}_{1}+\omega \mathrm{e}_{1} \wedge x$ for $(t, x) \in \mathbb{T} \times \partial \Omega$. Now define $v:=u-U$ and $p:=\mathfrak{p}$. Then $(u, \mathfrak{p})$ solves $(2.1)$ if and only if $(v, p)$ solves

$$
\left\{\begin{aligned}
\omega\left(\partial_{t} v+\mathrm{e}_{1} \wedge v-\mathrm{e}_{1} \wedge x \cdot \nabla v\right)-\Delta v-\lambda \partial_{1} v+\nabla p=f+\mathcal{N}(v) & \text { in } \mathbb{T} \times \Omega, \\
\operatorname{div} v=0 & \text { in } \mathbb{T} \times \Omega, \\
v=0 & \text { on } \mathbb{T} \times \partial \Omega, \\
\lim _{|x| \rightarrow \infty} v(t, x)=0 & \text { for } t \in \mathbb{T},
\end{aligned}\right.
$$

where

$$
\begin{aligned}
\mathcal{N}(v):= & \left(\mathcal{P}_{\perp} \alpha\right) \partial_{1} v-\omega\left(\partial_{t} U+\mathrm{e}_{1} \wedge U-\mathrm{e}_{1} \wedge x \cdot \nabla U\right) \\
& +\Delta U+\alpha \partial_{1} U-v \cdot \nabla v-U \cdot \nabla v-v \cdot \nabla U-U \cdot \nabla U
\end{aligned}
$$

Recall that $\mathcal{P}_{\perp} \alpha=\alpha-\lambda$. It thus remains to show existence of a solution to the nonlinear system (6.1).

Proof of Theorem 2.3. We define the function space

$$
\begin{aligned}
\mathcal{X}^{q}:= & \left\{v \in \mathrm{L}_{\mathrm{loc}}^{1}(\mathbb{T} \times \Omega) \mid\|v\|_{\mathcal{X}^{q}}<\infty\right\}, \\
\|v\|_{\mathcal{X}^{q}}:= & \omega\left\|\partial_{t} v+\mathrm{e}_{1} \wedge v-\mathrm{e}_{1} \wedge x \cdot \nabla v\right\|_{\mathrm{A}^{q}}+\left\|\nabla^{2} v\right\|_{\mathrm{A}^{q}} \\
& +\lambda\left\|\partial_{1} v\right\|_{\mathrm{A}^{q}}+\lambda^{1 / 2}\|v\|_{\mathrm{A}^{s_{1}}}+\lambda^{1 / 4}\|\nabla v\|_{\mathrm{A}^{s_{2}}},
\end{aligned}
$$


where $s_{1}=2 q /(2-q), s_{2}=4 q /(4-q)$ and

$$
\|h\|_{\mathrm{A}^{s}}:=\|h\|_{\mathrm{A}\left(\mathbb{T} ; \mathrm{L}^{s}(\Omega)\right)} .
$$

At first, we derive suitable estimates of $\mathcal{N}(v)$. For example, analogously to the proof of Proposition 3.1, we have

$$
\left\|\left(\mathcal{P}_{\perp} \alpha\right) \partial_{1} v\right\|_{\mathrm{A}^{q}} \leq\left\|\mathcal{P}_{\perp} \alpha\right\|_{\mathrm{A}(\mathbb{T} ; \mathbb{R})}\left\|\partial_{1} v\right\|_{\mathrm{A}^{q}} \leq \varepsilon\left\|\partial_{1} v\right\|_{\mathrm{A}^{q}} \leq \varepsilon \lambda^{-1}\|v\|_{\mathcal{X}^{q}}
$$

Moreover, since $\frac{2 q}{2-q} \leq 4 \leq \frac{3 q}{3-2 q}$, we can employ estimates (3.2) and (3.3) to obtain

$$
\|v \cdot \nabla v\|_{\mathrm{A}^{q}} \leq\|v\|_{\mathrm{A}^{4}}\|\nabla v\|_{\mathrm{A}^{4 q /(4-q)}} \leq c_{0}\|v\|_{\mathrm{A}^{2 q /(2-q)}}^{1-\theta}\|v\|_{\mathrm{A}^{3 q /(3-2 q)}}^{\theta}\|\nabla v\|_{\mathrm{A}^{4 q /(4-q)}}
$$

with $\theta=\frac{12-9 q}{2 q}$. By the Sobolev inequality we thus deduce

$$
\|v \cdot \nabla v\|_{\mathrm{A}^{q}} \leq c_{1} \lambda^{-1 / 4-(1-\theta) / 2}\|v\|_{\mathcal{X}^{q}}^{2-\theta}\left\|\nabla^{2} v\right\|_{\mathrm{A}^{q}}^{\theta} \leq c_{2} \lambda^{-(3 q-3) / q}\|v\|_{\mathcal{X}^{q}}^{2} .
$$

The remaining terms in $\mathcal{N}(v)$ can be estimated in a similar fashion, which results in

$$
\begin{aligned}
\|\mathcal{N}(v)\|_{\mathrm{A}^{q}} \leq c_{3}\left(\varepsilon \lambda^{-1}\|v\|_{\mathcal{X}^{q}}+\lambda^{-(3 q-3) / q}\|v\|_{\mathcal{X}^{q}}^{2}+\omega\left\|\frac{\mathrm{d}}{\mathrm{d} t} \alpha\right\|_{\mathrm{A}(\mathbb{T} ; \mathbb{R})}\right. \\
\left.+(\lambda+\omega+\varepsilon)\left(1+\lambda+\omega+\varepsilon+\|v\|_{\mathcal{X}^{q}}\right)\right) .
\end{aligned}
$$

Now consider the problem

$$
\left\{\begin{aligned}
\omega\left(\partial_{t} w+\mathrm{e}_{1} \wedge w-\mathrm{e}_{1} \wedge x \cdot \nabla w\right)-\Delta w-\lambda \partial_{1} w+\nabla \mathfrak{q}=f+\mathcal{N}(v) & \text { in } \mathbb{T} \times \Omega, \\
\operatorname{div} w=0 & \text { in } \mathbb{T} \times \Omega, \\
w=0 & \text { on } \mathbb{T} \times \partial \Omega
\end{aligned}\right.
$$

for given $v \in \mathcal{X}^{q}$. Due to estimate (6.2) and Theorem 2.2 there exists a unique velocity field $w \in \mathcal{X}^{q}$ and a pressure field $\mathfrak{q}$ with $\nabla \mathfrak{q} \in \mathrm{A}^{q}$ that satisfy (6.3) and the estimate

$$
\begin{aligned}
\|w\|_{\mathcal{X}^{q}} \leq & C_{1}\left(\|f\|_{\mathrm{A}^{q}}+\|\mathcal{N}(v)\|_{\mathrm{A}^{q}}\right) \\
\leq & c_{4}\left(\varepsilon+\varepsilon \lambda^{-1}\|v\|_{\mathcal{X}^{q}}+\lambda^{-(3 q-3) / q}\|v\|_{\mathcal{X}^{q}}^{2}+\omega\left\|\frac{\mathrm{d}}{\mathrm{d} t} \alpha\right\|_{\mathrm{A}(\mathbb{T} ; \mathbb{R})}\right. \\
& \left.+(\lambda+\omega+\varepsilon)\left(1+\lambda+\omega+\varepsilon+\|v\|_{\mathcal{X}^{q}}\right)\right) .
\end{aligned}
$$

We thereby obtain a solution map $\mathcal{S}: \mathcal{X}^{q} \rightarrow \mathcal{X}^{q}, v \mapsto w$ which is a self-mapping on the ball

$$
\mathcal{X}_{\delta}^{q}:=\left\{v \in \mathcal{X}^{q} \mid\|v\|_{\mathcal{X}^{q}} \leq \delta\right\}
$$

provided

$$
c_{4}\left(\varepsilon+\varepsilon \lambda^{-1} \delta+\lambda^{-(3 q-3) / q} \delta^{2}+\omega\left\|\frac{\mathrm{d}}{\mathrm{d} t} \alpha\right\|_{\mathrm{A}(\mathbb{T} ; \mathbb{R})}+(\lambda+\omega+\varepsilon)(1+\lambda+\omega+\varepsilon+\delta)\right) \leq \delta .
$$

Recall that $\rho \in\left(\frac{3 q-3}{q}, 1\right)$. Choosing $\delta:=\lambda^{\rho}$, one readily verifies that there is a constant $\kappa>0$ depending on $c_{4}$ such the condition above is satisfied with $\omega\left\|\frac{\mathrm{d}}{\mathrm{d} t} \alpha\right\|_{\mathrm{A}(\mathbb{T} ; \mathbb{R})} \leq \kappa \lambda^{\rho}, \varepsilon=\lambda^{2}$ and $\lambda_{0}$ sufficiently small. In the same way, one derives the estimate

$$
\left\|\mathcal{N}\left(v_{1}\right)-\mathcal{N}\left(v_{2}\right)\right\|_{\mathrm{A}^{q}} \leq c_{5}\left(\varepsilon \lambda^{-1}+\lambda+\omega+\varepsilon+\lambda^{-(3 q-3) / q}\left(\left\|v_{1}\right\|_{\mathcal{X}^{q}}+\left\|v_{2}\right\|_{\mathcal{X}^{q}}\right)\right)\left\|v_{1}-v_{2}\right\|_{\mathcal{X}^{q}},
$$

which ensures that $\mathcal{S}$ is a contraction on $\mathcal{X}_{\delta}^{q}$ with a similar choice of parameters. Finally, the contraction mapping principle yields the existence of a fixed point $v \in \mathcal{X}^{q}$ of $\mathcal{S}$, and hence of a solution $(v, p)$ to (6.1). Consequently, $(u, \mathfrak{p}):=(v+U, p)$ is a solution to $(2.1)$. 
Funding Open Access funding enabled and organized by Projekt DEAL.

\section{Compliance with Ethical Standards}

Conflict of Interest On behalf of all authors, the corresponding author states that there is no conflict of interest.

Open Access. This article is licensed under a Creative Commons Attribution 4.0 International License, which permits use, sharing, adaptation, distribution and reproduction in any medium or format, as long as you give appropriate credit to the original author(s) and the source, provide a link to the Creative Commons licence, and indicate if changes were made. The images or other third party material in this article are included in the article's Creative Commons licence, unless indicated otherwise in a credit line to the material. If material is not included in the article's Creative Commons licence and your intended use is not permitted by statutory regulation or exceeds the permitted use, you will need to obtain permission directly from the copyright holder. To view a copy of this licence, visit http://creativecommons.org/licenses/by/4.0/.

Publisher's Note Springer Nature remains neutral with regard to jurisdictional claims in published maps and institutional affiliations.

\section{References}

[1] Bruhat, F.: Distributions sur un groupe localement compact et applications à l'étude des représentations des groupes p-adiques. Bull. Soc. Math. Fr. 89, 43-75 (1961)

[2] Burenkov, V. I.: Extension of functions with preservation of the Sobolev seminorm. Trudy Mat. Inst. Steklov., 172:71-85, 1985. (English transl.: Proc. Steklov Inst. Math., 3:81-95, 1987)

[3] Edwards, R., Gaudry, G.: Littlewood-Paley and Multiplier Theory. Springer, Berlin (1977)

[4] Eiter, T., Kyed, M.: Time-periodic linearized Navier-Stokes equations: an approach based on Fourier multipliers. In: Particles in flows, Adv. Math. Fluid Mech., pp. 77-137. Birkhäuser/Springer, Cham (2017)

[5] Eiter, T., Kyed, M.: Estimates of time-periodic fundamental solutions to the linearized Navier-Stokes equations. J. Math. Fluid Mech. 20(2), 517-529 (2018)

[6] Farwig, R.: An $L^{q}$-analysis of viscous fluid flow past a rotating obstacle. Tohoku Math. J. 58(1), 129-147 (2006)

[7] Farwig, R., Neustupa, J.: On the spectrum of an Oseen-type operator arising from flow past a rotating body. Integral Eq. Oper. Theory 62, 169-189 (2008)

[8] Farwig, R., Neustupa, J.: Spectral properties in $L^{q}$ of an Oseen operator modelling fluid flow past a rotating body. Tohoku Math. J. 62(2), 287-309 (2010)

[9] Farwig, R., Sohr, H.: Generalized resolvent estimates for the Stokes system in bounded and unbounded domains. J. Math. Soc. Jpn 46(4), 607-643 (1994)

[10] Galdi, G., Kyed, M.: Time-periodic solutions to the Navier-Stokes equations in the three-dimensional whole-space with a non-zero drift term: Asymptotic profile at spatial infinity, volume 710 of Contemporary Mathematics, pp. 121-144. $01(2018)$

[11] Galdi, G., Sohr, H.: Existence and uniqueness of time-periodic physically reasonable Navier-Stokes flow past a body. Arch. Ration. Mech. Anal. 172(3), 363-406 (2004)

[12] Galdi, G. P.: On the motion of a rigid body in a viscous liquid: a mathematical analysis with applications. In Handbook of mathematical fluid dynamics, Vol. I, pp. 653-791. North-Holland, Amsterdam, (2002)

[13] Galdi, G. P.: An introduction to the mathematical theory of the Navier-Stokes equations. Steady-state problems. 2nd ed. Springer, New York (2011)

[14] Galdi, G.P.: On time-periodic flow of a viscous liquid past a moving cylinder. Arch. Ration. Mech. Anal. 210(2), 451-498 (2013)

[15] Galdi, G. P.: Viscous flow past a body translating by time-periodic motion with zero average. (2019)

[16] Galdi, G.P., Kyed, M.: A simple proof of $L^{q}$-estimates for the steady-state Oseen and Stokes equations in a rotating frame. Part I: Strong solutions. Proc. Amer. Math. Soc. 141(2), 573-583 (2013)

[17] Galdi, G.P., Kyed, M.: A simple proof of $L^{q}$-estimates for the steady-state Oseen and Stokes equations in a rotating frame Part II: Weak solutions. Proc. Am. Math. Soc. 141(4), 1313-1322 (2013)

[18] Galdi, G.P., Kyed, M.: Time-periodic flow of a viscous liquid past a body. In: Partial differential equations in fluid mechanics, volume 452 of London Math. Soc. Lecture Note Ser., pp. 20-49. Cambridge University Press, Cambridge (2018)

[19] Galdi, G. P., Kyed, M.: Time-periodic solutions to the Navier-Stokes equations. In: Handbook of Mathematical Analysis in Mechanics of Viscous Fluids, pp. 509-578. Springer, Cham (2018) 
[20] Galdi, G.P., Silvestre, A.L.: Strong solutions to the Navier-Stokes equations around a rotating obstacle. Arch. Ration. Mech. Anal. 176(3), 331-350 (2005)

[21] Galdi, G.P., Silvestre, A.L.: Existence of time-periodic solutions to the Navier-Stokes equations around a moving body. Pac. J. Math. 223(2), 251-267 (2006)

[22] Galdi, G.P., Silvestre, A.L.: On the motion of a rigid body in a Navier-Stokes liquid under the action of a time-periodic force. Indiana Univ. Math. J. 58(6), 2805-2842 (2009)

[23] Geissert, M., Hieber, M., Nguyen, T.H.: A general approach to time periodic incompressible viscous fluid flow problems. Arch. Ration. Mech. Anal. 220(3), 1095-1118 (2016)

[24] Grafakos, L.: Classical Fourier Analysis, 2nd edn. Springer, New York, NY (2008)

[25] Grafakos, L.: Modern Fourier Analysis, 2nd edn. Springer, New York, NY (2009)

[26] Heywood, J.G.: The Navier-Stokes equations: On the existence, regularity and decay of solutions. Indiana Univ. Math. J. 29, 639-681 (1980)

[27] Hishida, T.: Decay estimates of gradient of a generalized Oseen evolution operator arising from time-dependent rigid motions in exterior domains. Arch. Ration. Mech. Anal. 238(1), 215-254 (2020)

[28] Kaniel, S., Shinbrot, M.: A reproductive property of the Navier-Stokes equations. Arch. Rational Mech. Anal. 24, 363-369 (1967)

[29] Kozono, H., Nakao, M.: Periodic solutions of the Navier-Stokes equations in unbounded domains. Tohoku Math. J. 48(1), 33-50 (1996)

[30] Kyed, M.: Time-Periodic Solutions to the Navier-Stokes Equations. Technische Universität Darmstadt, Habilitationsschrift (2012)

[31] Kyed, M.: The existence and regularity of time-periodic solutions to the three-dimensional Navier-Stokes equations in the whole space. Nonlinearity 27(12), 2909-2935 (2014)

[32] Kyed, M.: Maximal regularity of the time-periodic linearized Navier-Stokes system. J. Math. Fluid Mech. 16(3), 523-538 (2014)

[33] Kyed, M.: A fundamental solution to the time-periodic Stokes equations. J. Math. Anal. Appl. 437(1), 708-719 (2016)

[34] Ladyženskaya, O.A.: Investigation of the Navier-Stokes equation for stationary motion of an incompressible fluid. Uspehi Mat. Nauk 14 (1959)

[35] Ladyženskaya, O.A.: The Mathematical Theory of Viscous Incompressible Flow, Second English Gordon and Breach Science Publishers, New York (1969)

[36] Leray, J.: Étude de diverses équations intégrales non linéaires et de quelques problèmes que pose l'hydrodynamique. J. Math. Pures Appl. 12, 1-82 (1933)

[37] Leray, J.: Sur le mouvement d'un liquide visqueux emplissant l'espace. Acta Math. 63(1), 193-248 (1934)

[38] Maremonti, P.: Existence and stability of time-periodic solutions to the Navier-Stokes equations in the whole space. Nonlinearity 4(2), 503-529 (1991)

[39] Maremonti, P.: Some theorems of existence for solutions of the Navier-Stokes equations with slip boundary conditions in half-space. Ric. Mat. 40(1), 81-135 (1991)

[40] Maremonti, P., Padula, M.: Existence, uniqueness and attainability of periodic solutions of the Navier-Stokes equations in exterior domains. Zap. Nauchn. Sem. S.-Peterburg. Otdel. Mat. Inst. Steklov. (POMI), 233 (Kraev. Zadachi Mat. Fiz. i Smezh. Vopr. Teor. Funkts. 27):142-182 (1996)

[41] Miyakawa, T., Teramoto, Y.: Existence and periodicity of weak solutions of the Navier-Stokes equations in a time dependent domain. Hiroshima Math. J. 12(3), 513-528 (1982)

[42] Morimoto, H.: On existence of periodic weak solutions of the Navier-Stokes equations in regions with periodically moving boundaries. J. Fac. Sci., Univ. Tokyo, Sect. I A 18, 499-524 (1972)

[43] Nguyen, T.H.: Periodic Motions of Stokes and Navier-Stokes Flows Around a Rotating Obstacle. Arch. Ration. Mech. Anal. 213(2), 689-703 (2014)

[44] Oseen, C.W.: Neuere Methoden und Ergebnisse in der Hydrodynamik. Akademische Verlagsgesellschaft M.B.H, Leipzig (1927)

[45] Prodi, G.: Qualche risultato riguardo alle equazioni di Navier-Stokes nel caso bidimensionale. Rend. Sem. Mat. Univ. Padova 30, 1-15 (1960)

[46] Prouse, G.: Soluzioni periodiche dell'equazione di Navier-Stokes. Atti Accad. Naz. Lincei Rend. Cl. Sci. Fis. Mat. Natur. (8) 35, 443-447 (1963)

[47] Serrin, J.: A note on the existence of periodic solutions of the Navier-Stokes equations. Arch. Rational Mech. Anal. 3, 120-122 (1959)

[48] Shibata, Y.: On the Oseen semigroup with rotating effect. In: Functional Analysis and Evolution Equations, pp. 595-611. Birkhäuser, Basel (2008)

[49] Silvestre, A.L.: Existence and uniqueness of time-periodic solutions with finite kinetic energy for the Navier-Stokes equations in $\mathbb{R}^{3}$. Nonlinearity 25(1), 37-55 (2012)

[50] Sohr, H.: The Navier-Stokes Equations. An elementary functional analytic approach. Birkhäuser, Basel (2001)

[51] Takeshita, A.: On the reproductive property of the 2-dimensional Navier-Stokes equations. J. Fac. Sci. Univ. Tokyo Sect. I 16(1970), 297-311 (1969)

[52] Taniuchi, Y.: On the uniqueness of time-periodic solutions to the Navier-Stokes equations in unbounded domains. Math. Z. 261(3), 597-615 (2009)

[53] Teramoto, Y.: On the stability of periodic solutions of the Navier-Stokes equations in a noncylindrical domain. Hiroshima Math. J. 13, 607-625 (1983) 
[54] Van Baalen, G., Wittwer, P.: Time periodic solutions of the Navier-Stokes equations with nonzero constant boundary conditions at infinity. SIAM J. Math. Anal. 43(4), 1787-1809 (2011)

[55] Yamazaki, M.: The Navier-Stokes equations in the weak- $L^{n}$ space with time-dependent external force. Math. Ann. 317(4), 635-675 (2000)

[56] Yudovich, V.: Periodic motions of a viscous incompressible fluid. Sov. Math., Dokl. 1, 168-172 (1960)

Thomas Eiter

Weierstrass Institute for Applied Analysis and Stochastics

Mohrenstr. 39

10117 Berlin

Germany

e-mail: thomas.eiter@wias-berlin.de and

Faculty of Science and Engineering Waseda University Tokyo

Japan

e-mail: kyed@aoni.waseda.jp

Mads Kyed

Flensburg University of Applied Sciences

Kanzleistraße 91-93

24943 Flensburg

Germany

e-mail: mads.kyed@hs-flensburg.de

(accepted: January 13, 2021; published online: February 11, 2021) 